Archive for

Organic Chemistry

Arkivoc 2020, part viii, 94-114

\title{
In-depth examination of the pterolactams behaviour in Lewis/Brönsted acid catalysis environment: Total isolation of the reaction products
}

\author{
Gina-Mirabela Dumitriu, ${ }^{a}$ Elena Bîcu, ${ }^{a}$ Benoît Rigo, ${ }^{b, c}$ Ján Moncol, ${ }^{d}$ Adam Daïch, ${ }^{* e}$ and Alina Ghinet*a,b,c \\ a 'Al. I. Cuza' University of Iasi, Faculty of Chemistry, Department of Organic Chemistry, Bd. Carol I nr. 11, \\ 700506 lasi, Romania \\ ${ }^{b}$ Ecole des Hautes Etudes d'Ingénieur, Laboratoire de Pharmacochimie, 13 rue de Toul, F-59046 Lille, France \\ ' Inserm, U995-LIRIC, CHRU de Lille, Faculté de Médecine-Pôle Recherche Université Lille, 2 Place Verdun, \\ F-59045 Lille Cedex, France \\ ${ }^{d}$ Department of Inorganic Chemistry, Faculty of Chemical and Food Technology, Slovak University of \\ Technology, SK-81237 Bratislava, Slovakia \\ ${ }^{e}$ Normandie Univ, UNILEHAVRE, URCOM, CNRS FR-3038, Le Havre, France. 25 rue Philippe Lebon, BP. 1123, \\ F-76063 Le Havre Cedex, France \\ Email: adam.daich@univ-lehavre.fr
}

Dedicated to our colleague and friend, Professor Benoît Rigo, on the occasion of his retirement.

Received 08-22-2020

Accepted $10-26-2020$

Published on line $11-30-2020$

\section{Abstract}

To gain some insights on the amidoalkylation process, we were interested in studying the product distribution when pterolactam was exposed to acids. The reactivity of pterolactam and its $N$-aryl derivatives towards TMSOTf or TfOH has been carefully examined in presence and absence of TMS-protected anilines. Under these conditions, the $\mathrm{N}$-acyliminium precursors showed high reactivity at both $\alpha$ - and $\beta$-positions of the nitrogen atom. In this context, these salts can induce cascade processes including Povarov type reaction among other transformations. In that way, novel quinolines and tetrahydro-quinolines, among other compounds, were isolated for the first time.

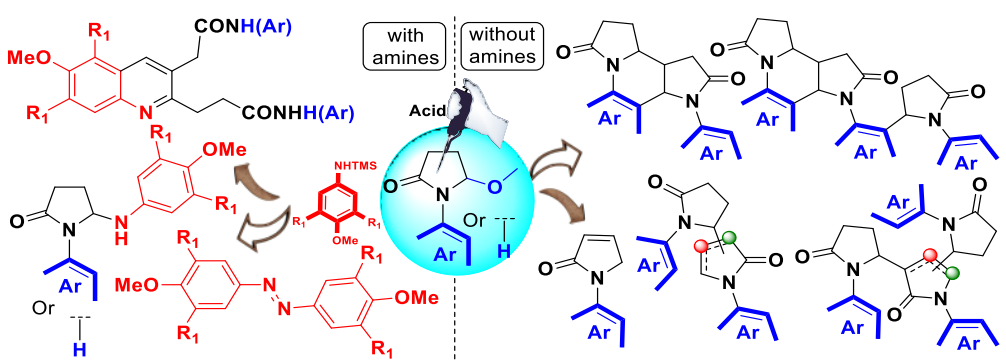

Keywords: Pterolactams, acid catalysis, $N$-acyliminiums, inter- and intra- $\alpha$-amidoalkylation, cascade process. 


\section{Introduction}

Coupling reactions between nucleophiles and linear $\mathrm{N}$-acyliminium ions (NAI) usually result in low to medium regio- and stereoselectivity, generally due to the high degrees of freedom of cationic precursor species. The use of cyclic NAl, having lower degrees of freedom, allows better control of the regio- and diastereoselectivity of the amido-alkylation process, which allowed the synthesis of natural and unnatural aza-heterocyclic complex molecules ${ }^{1-6}$ While the $\mathrm{C}-$ heteroatom bond formation proved to be scarce, ${ }^{7}$ the $\mathrm{C}-\mathrm{C}$ bond forming reactions, have extensively investigated, and are still of interest.

Of particular interest, the cyclic NAI are characterised by great structural variability, and among them two major families can be distinguished: the more stable aromatic ions of type (I) and the more reactive cyclic ions of type (II), which may themselves be endocyclic ${ }^{8}$ or exocyclic ${ }^{9,10}$ (Scheme 1 ). In the latter case, the acyl $\mathrm{C}=0$ group is attached to the $N$ atom instead of the R group, however, these exocyclic NAl are not shown in Scheme 1 to simplify the Scheme.
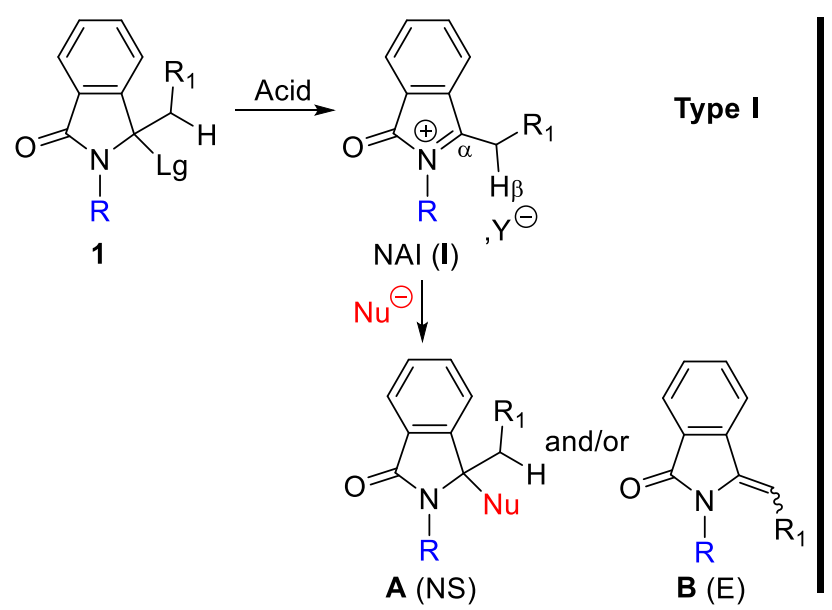

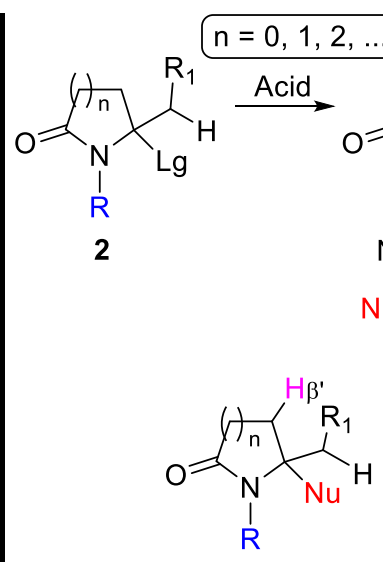

A (NS)

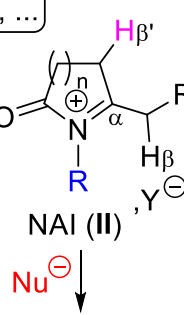

N-Acyliminium Intermediate: NAI Leaving group: $\mathrm{Lg}$

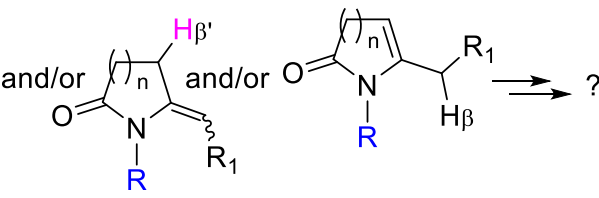

B (E)
Type II

C (E)

Scheme 1. Different reactivity modes of cyclic NAI generated form lactams $\mathbf{1}$ and $\mathbf{2}$ according to nucleophilic substitution (NS) vs. elimination (E).

In that context and according to Scheme 1 , in addition to the classical nucleophilic substitution (NS) reaction products $\mathbf{A}$, other products (B) can be obtained by $\mathrm{H}_{\beta}$ elimination (E). In the case of NAl of type (II), $\mathrm{H}_{\beta^{\prime}}$ elimination can occur giving endocyclic enamidones $\mathrm{C}^{11}$ able to react further in the reaction medium forming complexes molecules. In order to deepen this question, our aim beyond the importance of the products that will be obtained, is to study the overall behavior of NAI (II) generated in an acid environment in the absence or presence of an external nucleophile. Due to the poor literature data on this area, the isolation of all the reaction products will be systematically undertaken.

\section{Results and Discussion}

During our previous studies on pterolactams $\mathrm{s}^{12-14}$ we have already explored the reactivity of pterolactam derivatives of type $\mathbf{1}\left(\mathrm{R}=\mathrm{H}, \mathrm{Me}, \mathrm{CH}_{2} \mathrm{Ar}\right.$ ) with anilines $\mathbf{3}$ or $\mathrm{N}$-trimethylsilylanilines $\mathbf{4}$ by using triflic acid (TfOH) or trimethylsilyl triflate (TMSOTf) as a catalyst (Scheme 2). The results showed that $\alpha$-aminoalkylation reaction is clean and the use of TMS-protected amines 4 gives higher yields (55\% instead of $40-50 \%$ with anilines 3 ) 
under shorter reaction time (Scheme 2$).{ }^{15}$ In addition, via this reaction, compounds $\mathbf{5 a , b}$, and the potential farnesyltransferase (FTase) inhibitors 6A,B could be synthesized (Scheme 2). ${ }^{16-18}$ Of interest, attempts to generalize this amination method showed that the process is very sensitive to the acidity of the reaction medium and that addition, changing the concentrations and/or the nature of the acid used as catalyst completely modifies the reaction profile.

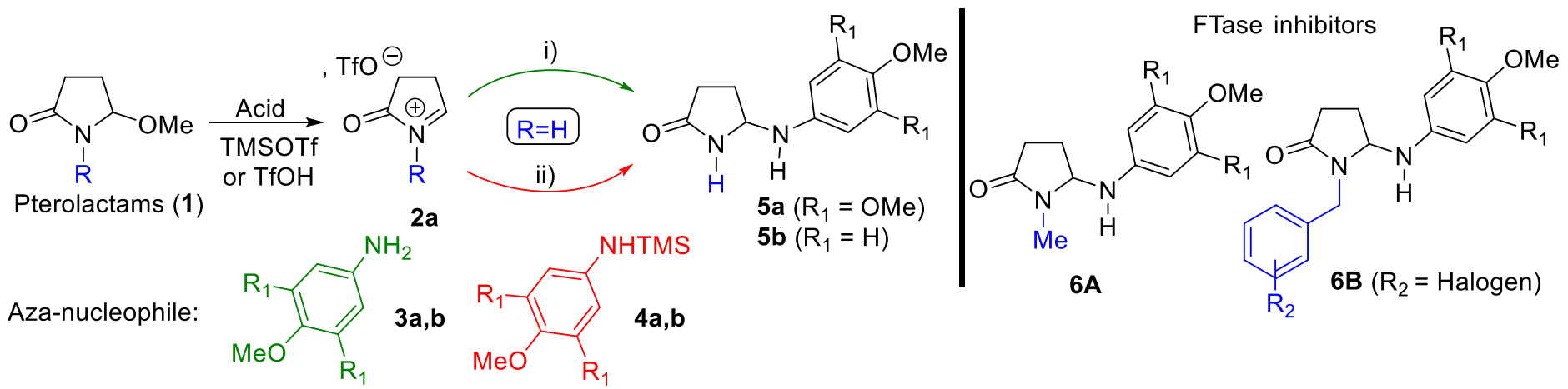

Reaction conditions: i) TfOH (10-15 mol\%), $3, \mathrm{CH}_{2} \mathrm{Cl}_{2}, 50{ }^{\circ} \mathrm{C}, 24-48 \mathrm{~h}(40-50 \%)$; ii) TMSOTf (5 mol\%), $4, \mathrm{CH}_{2} \mathrm{Cl}_{2}, 50{ }^{\circ} \mathrm{C}, 8 \mathrm{~h}(55 \%)$.

Scheme 2. $\alpha$-Aminoalkylation of cyclic NAI 2a into N,N-aminals 5-6 from pterolactams 1 under acid catalysis.

We began the study by re-examining the reaction of pterolactam 1a with $N$-trimethylsilylamine $\mathbf{4 a}$ under different acidic conditions by using a double amount of TMSOTf catalyst (10 mol\%) that we have used in an earlier report in 5 mol\%. ${ }^{15}$ Thus after heating for $14 \mathrm{~h}$ at $50{ }^{\circ} \mathrm{C}$, the $\mathrm{N}, \mathrm{N}$-aminal product $5 \mathrm{a}^{15}$ was isolated in only $10 \%$ yield after chromatography separation. The latter compound was accompanied by many new compounds identified as the quinolines $7 \mathrm{a}, \mathbf{8} \mathbf{a}$ and $9 \mathrm{a}$ in $0.15 \%$ to $2 \%$ yields, in addition to the amine $10 \mathrm{a}$ (trance) and the azo-derivative 11a in $1 \%$ yield (Scheme 3 ).

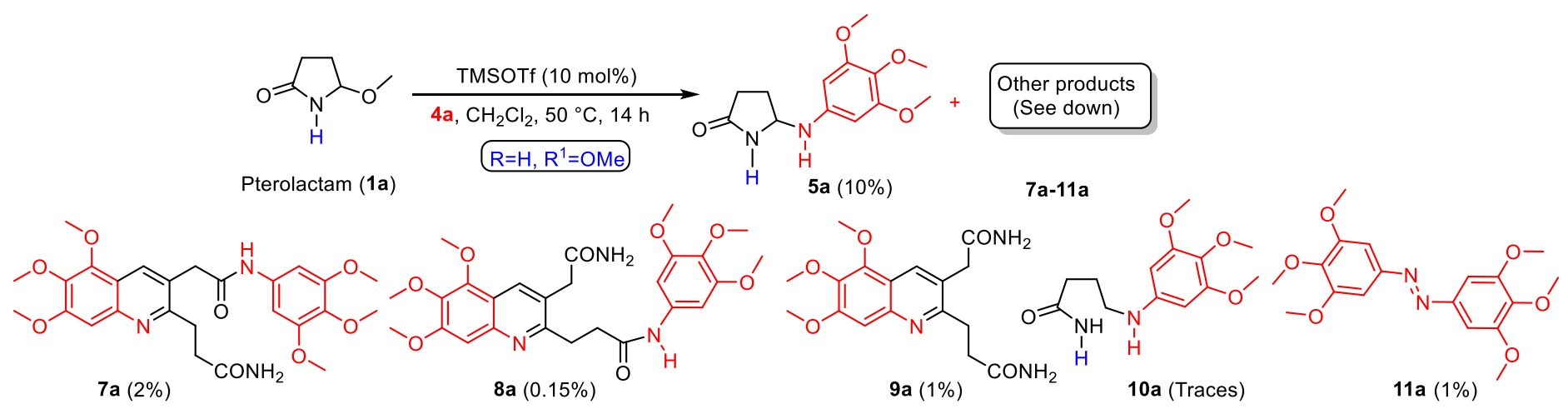

Scheme 3. $\alpha$-Aminoalkylation of 1a under TMSOTf catalysis.

Considering the literature on slightly related transformations, the formation of compounds $\mathbf{5 a}$ and $\mathbf{7 a - 1 1 a}$ can be explained as described in Scheme 4. Thus, pterolactam 1a under acidic conditions can provide the NAI $2 \mathbf{a}$ which gave the enamide $12 \mathbf{a}$ by functional equilibration, ${ }^{19-23}$ and also $N, N$-aminal derivative 5 a by $\alpha$ aminoalkylation ${ }^{15}$ when reacted with TMS aniline $\mathbf{4 a}$. All the three compounds $\mathbf{2 a}$, $\mathbf{5 a}$, and $\mathbf{1 2 a}$ are in equilibrium in the acid medium. Ring opening of $N, N$-aminal derivative 5 a furnished aromatic imine $\mathbf{L}$, which under acid catalyzed Povarov type reaction (aza-Diels-Alder reaction) ${ }^{24-30}$ with enamide 12a provided tetrahydroquinoline intermediate $\mathbf{M}$. The latter after lactam ring cleavage ${ }^{31-34}$ furnished dihydroquinoline $\mathbf{N}$ 
whose air oxidation provided the isolated quinoline product 9a (1\%). Interestingly, no lactam cleavage was reported in related piperidinoquinolones. ${ }^{24}$ Then the trans-amidification at the primary amides' sites of quinoline $9 \mathrm{a}$ by TMS-protected amine $\mathbf{4 a}$, ultimately yielded dissymmetrical quinoline diamides $7 \mathbf{a}(2 \%)$ and $8 \mathrm{a}$ $(0.15 \%)$. As for azo-benzene 11a, it was formed by a standard oxidative dimerization of the starting aniline obtained by TMS-amine deprotection under acidic conditions. ${ }^{31,34}$ Finally, the formation of 10 a, detected in the reaction medium only in trace amounts, could be due to the in-situ imine reduction of $\mathbf{L}$ by proton transfer from the fused tetrahydroquinoline $\mathbf{M}$ and/or dihydroquinoline $\mathbf{N}$ intermediates. ${ }^{24-30}$

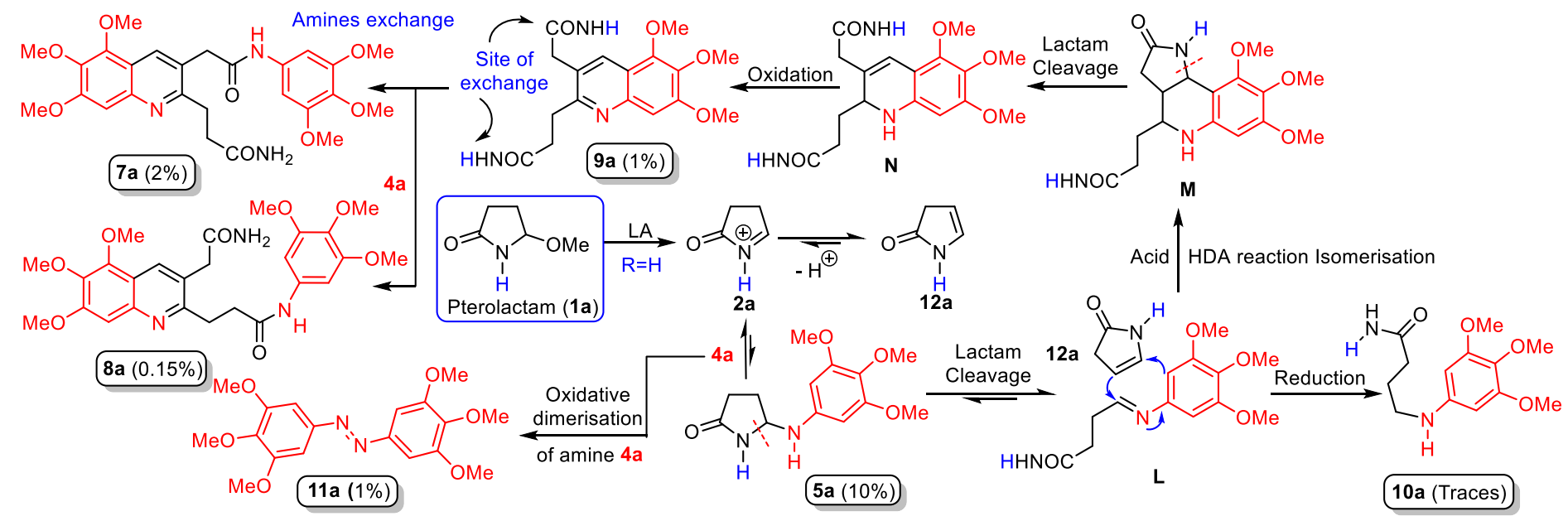

Scheme 4. Postulated mechanism for $\alpha$-aminoalkylation of $1 \mathrm{a}(\mathrm{R}=\mathrm{H})$ leading to compounds $\mathbf{5 a}, \mathbf{7 a - 1 1 a .}$

Quinoline scaffold is present in many biologically important natural and unnatural compounds, ${ }^{37-42}$ and the formation of these systems with substituents at privileged positions could lead to new compounds with important biological properties. Interestingly, many approaches on the synthesis of quinolines and their application domains were described in numerous reviews, ${ }^{43,44}$ and to the best of our knowledge, neither pterolactam 1a nor its $N$-aryl derivatives were used in these approaches to date. Thus, the control of this new synthesis in exploitable manner, in terms of yields and limitation of by-products, could render this methodology very attractive.

In order to collect additional information on the scope of this new quinoline synthesis, two $N$-aryl lactams, $\mathbf{1 b}\left(\mathrm{R}_{2}=\mathrm{Ph}\right)$ and $\mathbf{1 c}\left(\mathrm{R}_{2}=1,3-\mathrm{Cl}_{2} \mathrm{Ph}\right)$ were chosen. These substrates $\mathbf{1} \mathbf{b}, \mathbf{c}$ obtained by $\mathrm{N}$-arylation of pterolactam (1a) under Buchwald type coupling reaction, ${ }^{35}$ were submitted to reaction with TMS-protected anilines $4 \mathbf{b}$ and 4a (Scheme 2), respectively, under acidic conditions (Scheme 5).

As highlighted in Scheme 5, the reaction between $N$-phenylpterolactam (1b, R $\left.=P h ; R_{2}=H\right)$ and TMS-aniline 4b, was complete after heating for $96 \mathrm{~h}$ in the presence $20 \mathrm{~mol} \%$ of $\mathrm{TfOH}$, where only three products were isolated. The trisubstituted quinoline $\mathbf{9 b}$ was obtained as a major compound in $30 \%$ yield, accompanied by amino-amide $\mathbf{1 0 b}$ in $5 \%$ yield and the azo-derivative $\mathbf{1 1 b}$ in only $1 \%$ yield. No $N$-phenyl $N, N$-aminal $\mathbf{5 b}$, or dissymmetrical quinoline amides analogs to $7 \mathbf{a}$ and $\mathbf{8 a}$ (Scheme 3 ) could be detected in the reaction mixture. However, the reaction between of $N$-(3,5-dichlorophenyl)-pterolactam (1c) and TMS-aniline $4 \mathrm{a}$ in the presence of $\mathrm{TfOH}$ required the use $\mathrm{AcOH}$ as a solvent, because of the high melting point of the reaction mixture The reaction showed the same profile as for $\mathbf{1 b}$ (Scheme 5 ) where the trisubstituted quinoline $\mathbf{9 c}$ was isolated in good yield (53\%), accompanied with only insignificant amounts of amino-amide $8 \mathrm{c}$ (traces) and $18 \%$ of the azo-compound 11c. Also, no compounds such as $\mathbf{5 c}, \mathbf{7 c}$ and $\mathbf{8 c}$ were isolated from the reaction mixture. From these results, it seems that $N$-aryl- $N, N$-aminal moiety such as in $\mathbf{5 b}$ or $\mathbf{5 c}$ is unstable in acid medium. This is 
beneficial and favors the reaction profile to give quinolines. The quinolines $9(9 \mathrm{c}>>9 \mathrm{~b}>>9 \mathrm{9})$ and azocompounds 11 (11c $>>11 b \approx 11 a)$ was obtained instead of the formation of amino-amides $\mathbf{1 0}$ which becomes totally marginal. Finally, the $\mathrm{N}$-aryl amides $\mathbf{9 b , c}$ were not subjected to the trans-amidification observed in case of reagent 1a.
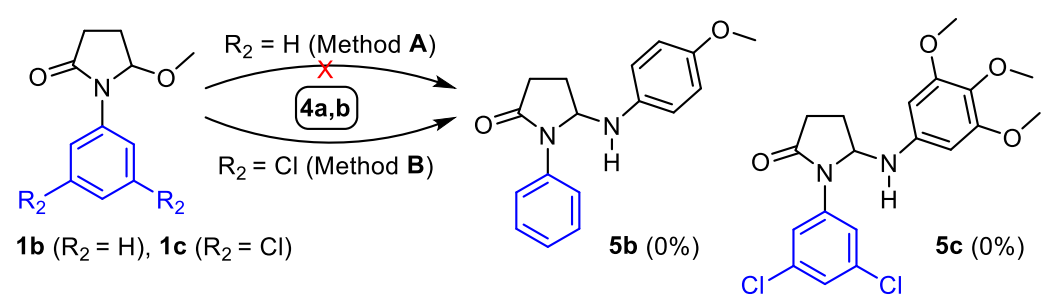

Reactions conditions:

Method $\mathbf{A}\left(\mathrm{R}_{2}=\mathrm{H}\right): \mathbf{4 b}$, TfOH $(20 \mathrm{~mol} \%), \mathrm{CH}_{2} \mathrm{Cl}_{2}, 40^{\circ} \mathrm{C}, 96 \mathrm{~h}$. Method $\mathbf{B}\left(\mathrm{R}_{2}=\mathrm{Cl}\right): 4 \mathrm{a}$, TfOH (10 mol\%), AcOH (10 mol\%), $\mathrm{CH}_{2} \mathrm{Cl}_{2}, 40^{\circ} \mathrm{C}, 96 \mathrm{~h}$.

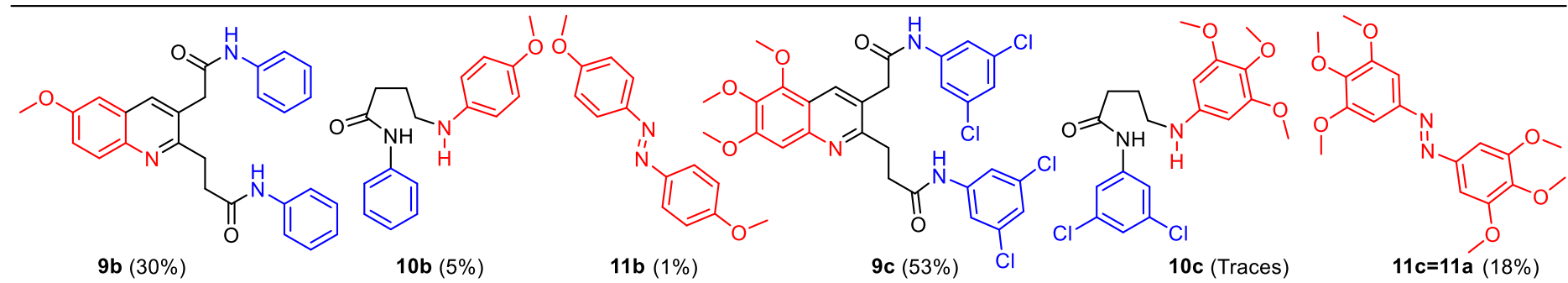

Scheme 5. Influence of $N$-aryl substituent on reaction of $\mathbf{1 b}, \mathbf{c}$ with amines $\mathbf{4 a ,} \mathbf{b}$ under acid catalysis.

Furthermore, in the hope of obtaining $N$-aryl- $N, N$-aminal products, parallel investigations were done on the Buchwald reaction between $N, N$-hemi-aminal 5 a and 1,3-dichloro-5-iodobenzene (13a). As illustrated in Scheme 6 , the reaction realized under the conditions published earlier, ${ }^{35}$ provides regiochemically the expected product $\mathbf{5 c}$ in $72 \%$ yield. This is in accordance with our report ${ }^{35}$ and demonstrates in the same time that $N, N$-aminal functionality is stable in alkaline media compared with acidic one. ${ }^{45,46}$

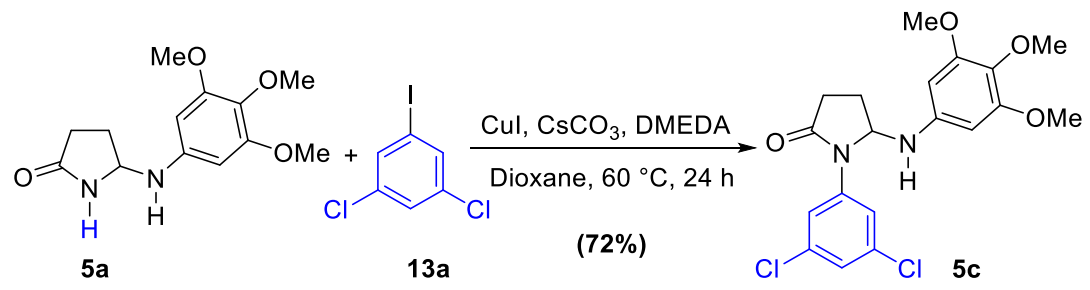

Scheme 6. Synthesis of $N, N$-aminal $5 c$ under coupling $N$-arylation conditions.

In all $\alpha$-aminoalkylation reactions of $N$-aryl pterolactams 1 with TMS-protected anilines described above, very few amounts of unknown by-products were also observed by ${ }^{1} \mathrm{H}-\mathrm{NMR}$ studies. They do not present elements of the used aniline as copartner. In an attempt to identify these compounds, obtain them in measurable amounts, and understand the possible mechanism allowing their formation, the reaction was realized without TMS-anilines. Also, to determine the influence of acid amount on the reaction profile, the NAI precursors $\mathrm{N}$-aryl pterolactams 1 were stirred in $\mathrm{CH}_{2} \mathrm{Cl}_{2}$ under different conditions of temperature and amount of TMSOTf (see Scheme 8 and Table 1). 


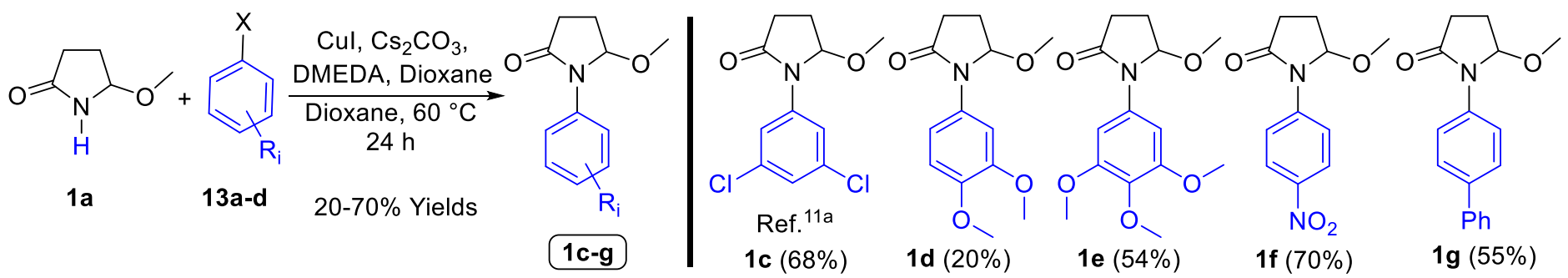

Scheme 7. Production of $N$-arylpterolactams 1c-g necessary for next investigations by $N$-arylation coupling.

In that line, selected $\mathrm{N}$-aryl pterolactams $1 \mathrm{c}-\mathrm{g}$ with different substituents on the phenyl group necessary for our next investigations in acid medium, were firstly prepared in good yields from pterolactam $1 \mathrm{a}(\mathrm{R}=\mathrm{H})$ as highlighted in Scheme 7.

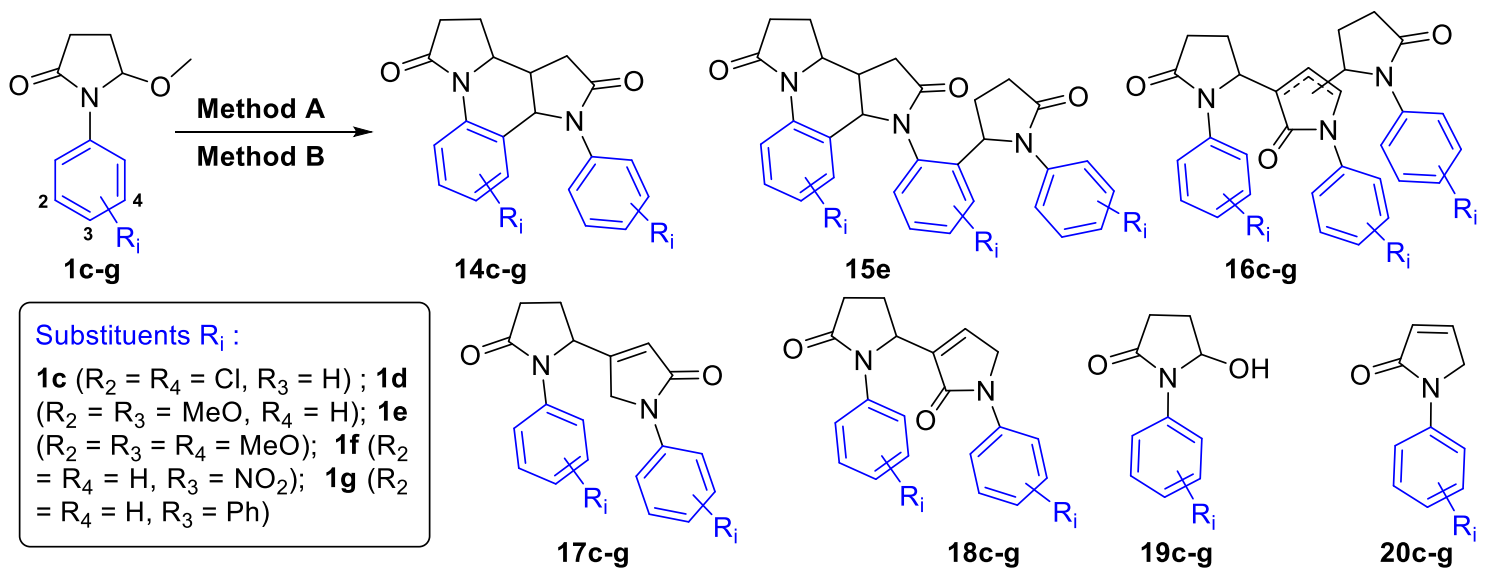

Scheme 8. Transformation of pterolactams $1 \mathrm{c}-\mathrm{g}$ without amines under acid catalysis. Reactions conditions: Method A: TMSOTf (5 mol\%), $\mathrm{CH}_{2} \mathrm{Cl}_{2}, 20-50{ }^{\circ} \mathrm{C}, 2-4$ days. Method B: TMSOTf (50 mol\%), $\mathrm{CH}_{2} \mathrm{Cl}_{2}, \mathrm{rt}$., 1-2 h.

Table 1. Casting (\%) of formed products stemming from the evolution of $\mathbf{1 c}-\mathbf{g}$ in acid medium as in Scheme 8.

\begin{tabular}{|c|c|c|c|c|c|c|c|}
\hline Product $^{a}$ & 14 & 15 & $16\left(16^{\prime}\right)$ & 17 & 18 & 19 & 20 \\
\hline \multirow[t]{2}{*}{ SM: $1 c^{b}$} & $30 \%^{c}$ & - & - & - & - & - & - \\
\hline & $5 \%^{d}$ & - & $90 \%$ & - & - & - & - \\
\hline \multirow[t]{2}{*}{ SM: 1d } & $42 \%$ & - & - & - & - & $40 \%$ & - \\
\hline & $52 \%(7 \%)^{\mathrm{e}}$ & - & - & - & - & Traces & - \\
\hline \multirow[t]{2}{*}{ SM: 1 e } & $35 \%$ & Traces & - & - & - & $40 \%$ & - \\
\hline & $60 \%$ & $24 \%$ & - & - & - & $5 \%$ & - \\
\hline \multirow[t]{2}{*}{ SM: $1 \mathbf{f}$} & - & - & - & - & - & - & - \\
\hline & - & - & - & $25 \%$ & $5 \%$ & - & - \\
\hline \multirow[t]{2}{*}{ SM: 1g } & - & - & - & - & - & - & - \\
\hline & $50 \%^{f}$ & - & $10 \%(8 \%)^{\mathrm{e}}$ & - & $7 \%$ & $2 \%$ & $2 \%$ \\
\hline
\end{tabular}

a Yields of isolated products. ${ }^{b}$ SM: Starting material: pterolactams 1c-g. ${ }^{c}$ Yields obtained from Method A. ${ }^{d}$ Yields (in bold) obtained from Method B. ${ }^{\text {e }}$ Other stereoisomer. ${ }^{f}$ Mixture containing two stereoisomers. 
The $\mathrm{Cu}(\mathrm{I})$-catalyzed $\mathrm{Csp}^{2}-\mathrm{N}$ coupling process conditions used in Scheme 7 are same as for products $\mathbf{1 b}$ and $\mathbf{5 c}$ (Scheme 6). ${ }^{35}$ The low yield isolated in the case of product $\mathbf{1 d}$ reflects mainly the purification difficulties encountered during the reaction work up.

From the results reported in Table 1, it appears that the use of $5 \mathrm{~mol} \%$ of $\mathrm{TMSOTf}$ in $\mathrm{CH}_{2} \mathrm{Cl}_{2}$ at $20-50{ }^{\circ} \mathrm{C}$ for 2-4 days (Method A), or reactions in $\mathrm{CH}_{2} \mathrm{Cl}_{2}$ at room temperature, with 50 mol\% of TMSOTf for 1-2 hours (Method B), lead to different reaction profiles (Scheme 8). In the case of Method A, with low amount of catalyst, the reaction with 1c-e gave a mixture of $N$-aryl polyhydropyrroloquinolines (14c-e) in $30-42 \%$ yield and 5-hydroxy-1-arylpyrrolidin-2-ones (19c-e) in 40\% yields, except for pterolactam derivative 1c. The formation of tricyclic systems 14c-e can be explained by the already reported dimerization process for related compounds via an interesting intermolecular $\pi$-cationic cyclisation. ${ }^{24,47-50}$ As for hydroxyl lactams $19 \mathrm{c}-\mathrm{g}$, they were generated by hydrolysis of the unreacted NAI intermediates during the reaction work up. Only traces of compounds $\mathbf{1 5 e}$ were detected, and no reaction in cases of methoxy lactams $\mathbf{1 f , g}$ was observed when using Method A.

On the other hand, as outlined in Table 1, treatment of pterolactams 1c-e using 50 mol\% of TMSOTf according to the Method B, provides mainly cyclized dimers $\mathbf{1 4}$ and also trimers $\mathbf{1 5}$ formed from reaction of $\mathbf{1 4}$ with aryl pterolactam 1, with very low amounts of hydroxyl lactams 19. Interestingly, starting from reactants 1c and $\mathbf{1 g}$ also uncyclized trimers $\mathbf{1 6}$ formed from the reaction of $\mathbf{1 7}$ and $\mathbf{1 8}$ were isolated in $90 \%$ and cumulated $18 \%$, respectively (Scheme 8, Table 1 ). The reactions proceeded with high yields which are globally inverted from $1 \mathrm{c}$ to $1 \mathrm{e}$. In addition, the short reaction time (1-2 $\mathrm{h}$ for completion determined by TLC) and the room temperature needed under these conditions, outline the importance of the acid concentration for the kinetic, the selectivity and the yields of these reactions.

In particular with $\mathrm{N}$-( $p$-nitrophenyl)pterolactam 1f, the reaction leads to only uncyclized dimers $17 \mathbf{f}(25 \%)$ and $18 f(5 \%)$, whereas starting from $N$-(p-diphenyl)pterolactam $1 \mathrm{~g}$, a plethora of compounds including monomers $19 f(2 \%)$ and $20 f(2 \%)$, dimer $18 f(7 \%)$ and cyclized dimer $\mathbf{1 4 g}(50 \%)$ were obtained. In addition, the latter compounds were accompanied with uncyclized trimer $16 \mathrm{~g}(10 \%)$ and its regioisomer 16 ' $\mathrm{g}(8 \%)$ with $14 \mathrm{~g}$ obtained as mixture of two isomers (see Table 1). Thus, it appears that the strong electro-withdrawing effect of the nitro group on NAI precursor 1 f aborted the $\pi$-cationic cyclization whatever the dimer resulting from the $\alpha$-amidoalkylation. This result is contradictory to that of the bis-phenyl group which exerts non-selectivity on the reaction profile.

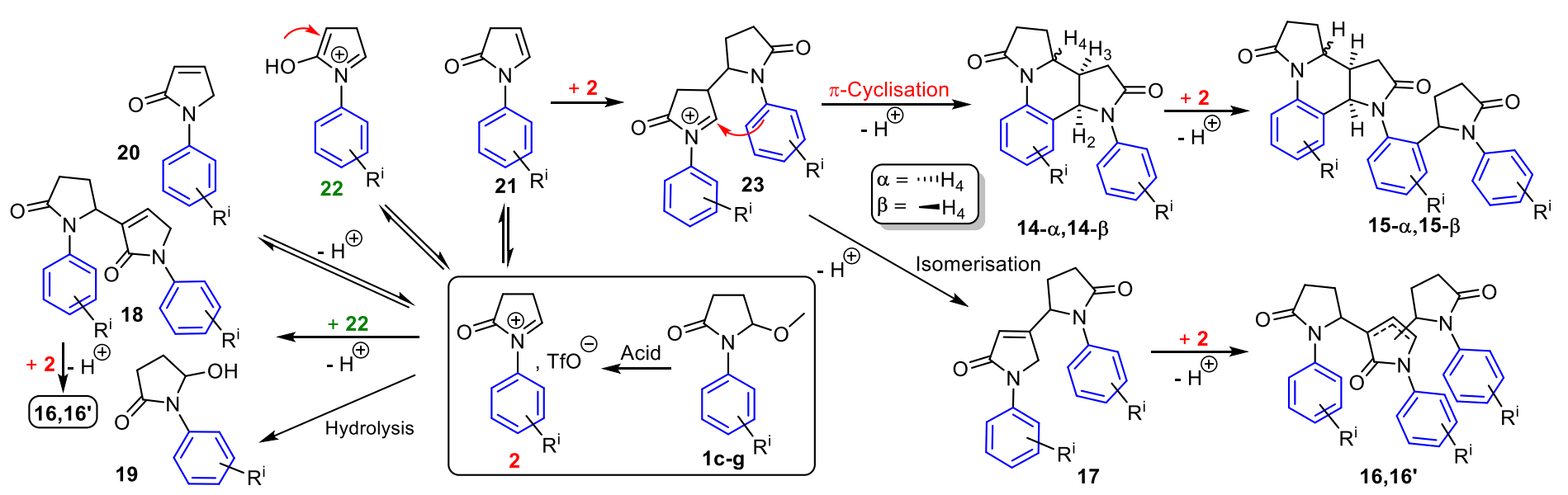

Scheme 9. Plausible mechanism for the formation of 14-20 from pterolactams $1 \mathrm{c}-\mathrm{g}$ ( $\mathrm{R}^{\mathrm{i}}$ are substituents). 
The pioneer work of Speckamp's group in the chemistry of NAI has demonstrated that $\omega$-alkoxy lactams treated with an acid decomposed into several kinds of compounds. Some of them are mentioned in the general mechanism we proposed for the production of derivatives 14-19 (Scheme 9). ${ }^{22,23,32,33,51-53}$ Structurally speaking, identification of the obtained products and their stereochemistry in particular of the cyclic dimers $\mathbf{1 4}$ and the trimers 15 and 16 were determined by MS, NMM NOESY experiments, and crystallographic analysis, in addition to traditional physico-chemical methodologies.

On the basis of the contributions of Speckamp's group and others in this area, ${ }^{22,23,32,33,51-53}$ we identified easily some derivatives such as $\omega$-carbinol lactams 19 and enamides $\mathbf{2 0}$. They, respectively, were formed by a facile hydrolysis or isomerization of the NAI of type $\mathbf{2}$ in the acid medium. The formation of dimers $\mathbf{1 7}$ can be explained by steps-sequence involving the formation of enamides $\mathbf{2 1}$ (never isolated in our case), their reaction with NAI $\mathbf{2}$ followed by deprotonation of intermediate salt $\mathbf{2 3}$, and their spontaneous isomerization. For dimers 18, the same sequence can be considered, being preceded by the isomerization of the cation $\mathbf{2}$ into 22 which now acted as carba-nucleophile. Some of these uncyclized dimers scaffolds are described to be unstable depending on the acid used as catalyst. ${ }^{32,33,51,53}$

In another way, the NAl intermediates 23 provided, via $\pi$-cationic cyclization, the cyclic dimers 14 as two stereoisomers $14-\alpha$ and $14-\beta$. In certain cases, these products were separated by chromatography on silica gel column. Finally, the cyclized trimers $15-\alpha$ and $15-\beta$, for which no-equivalent structure was published yet in the literature, were obtained by regioselective $\alpha$-amidoalkylation reaction of $14-\alpha$ and $14-\beta$ with NAI 2 as nucleophilic and electrophilic partners, respectively.

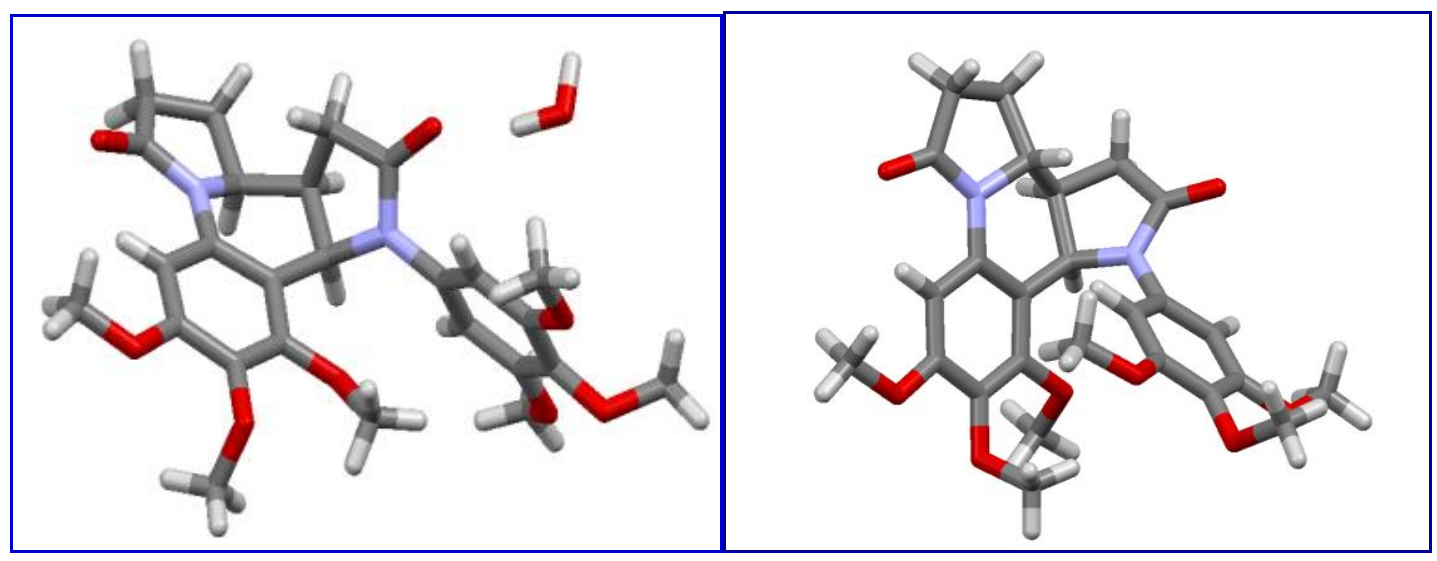

Part I (Stick drawing of 14e- $\alpha$ )

Part II (Stick drawing of $14 \mathrm{e}-\beta$ )

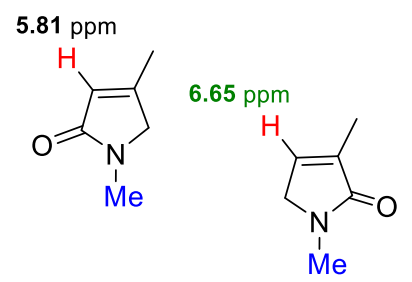

24A

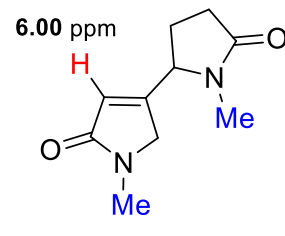

25A

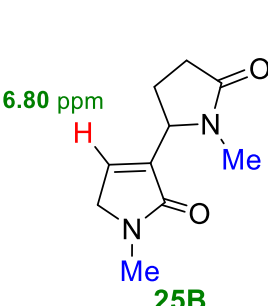

25B

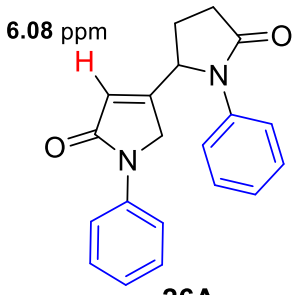

26A

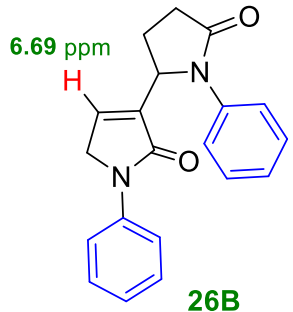

26B

Figure 2. X-ray structure of $14 \mathrm{e}-\alpha$ (Part I) and $14 \mathrm{e}-\beta$ (Part II) crystallized, respectively, with one molecule of water or alone. Chemical shifts of the ethylenic proton for 24-26(A,B) extracted from the literature (Part III).

Thanks to the careful NMR interpretation, and especially the chemical shifts of the ethylenic proton extracted from the literature for compounds 24-26(A,B) (Figure 2), ${ }^{22,23,48,52}$ associated with ${ }^{2} \mathrm{D}$ NMR, have 
constituted important backgrounds for the identification of a big part of products 14-20 (Schemes 8, 9). On the other side, the X-ray crystal structure of the representative cyclized major isomer $14 \mathbf{e}-\alpha$ and minor isomer 14e- $\beta$ was realized; ${ }^{54}$ the three contiguous angular protons $\mathrm{H}_{2}, \mathrm{H}_{3}$ and $\mathrm{H}_{4}$ (as outlined in Scheme 9) are in the same side for the isomer $14 \mathrm{e}-\alpha$ while $\mathrm{H}_{4}$ in the opposite side to $\mathrm{H}_{2}, \mathrm{H}_{3}$ for the isomer $14 \mathrm{e}-\beta$ (Figure 2). This stereochemistry relationship between the three angular protons is transferred to the high-ranking compounds, the trimers $15-\alpha$ and $15-\beta$, and that was confirmed by the rather simple ${ }^{1} \mathrm{H}$ NMR of trimer $15 \mathrm{e}-\alpha$ for example. Finally, the relative configuration of the contiguous three tertiary stereocenters are found in the structure of natural Incargranine- $\mathrm{B}\left(\mathrm{H}_{2-4} \text { and } \mathrm{H}_{3-4} \text { all cis }\right)^{55}$ and aglycon of Incargranine- $\mathrm{B}\left(\mathrm{H}_{2-3}\right.$ cis and $\mathrm{H}_{3-4}$ trans) whose syntheses were described very recently from an efficient $\mathrm{Cu}(\mathrm{OTf})_{2}$-catalyzed cascade cyclization reaction of simple homopropargylic amines. ${ }^{56}$ Noteworthy, taking into account that crystals of trimers $16 \mathrm{c}$ and $16 \mathrm{~g} / \mathbf{1 6}$ 'g suitable for an X-ray structure confirmation cannot be obtained, and these compounds degrade rapidly in the NMR solvents tried. Their structure was however deduced from their ${ }^{1} D$ NMR which highlights no similarity with the cyclized trimer $15 \mathbf{e}$.

\section{Conclusions}

Beyond the utility of the amidoalkylation reaction of NAl for the preparation of $\mathrm{N}, \mathrm{N}$-aminals which can possess desirable FTase activities, ${ }^{15}$ we were interested in this contribution to isolate all the products stemming from this reaction conducted with pterolactms under acidic conditions. Surprisingly, in addition to conventional products, we showed that this reaction gives rise to several transformations, sometimes complex, in which the products obtained in small quantities were often ignored in the literature.

Concisely, the reactivity of pterolactam and its corresponding $N$-aryl derivatives towards TMSOTf and TfOH has been investigated in presence and absence of TMS-protected anilines as aza-nucleophiles. The first set of amines lead to original functionalized quinolines by cascade reactions. The latter include the formation of enamides and $\mathrm{N}, \mathrm{N}$-aminals via $\mathrm{NAl}$, ring opening of $\mathrm{N}, \mathrm{N}$-aminals into aldimines which are reduced by proton transfer or embarked in an interesting Povarov type reaction (aza-Diels-Alder). The quinolines were finally reached by lactam cleavage, oxidation followed by a trans-amidification.

On the contrary, without amines, the starting pterolactams under similar acidic conditions generate the classical NAI. The latter NAI unstable undergo hydrolysis in certain cases, or deprotonation into corresponding enamides. These species by $\alpha$-amidoalkylation furnish cyclized or uncyclized dimers via another intermolecular or intramolecular $\pi$-cationic cyclisation. These reactions generate the creation of three angular tertiary stereocenters present in different complex alkaloids, whose stereochemical integrity rests intact in trimers obtained ultimately by another $\alpha$-amidoalkylation with the initially cation. Finally, the relative stereochemistry of the stereocenters being formed was confirmed by X-ray analysis.

\section{Experimental Section}

General. Starting materials are commercially available and were used without further purification (suppliers: Carlo Erba Reagents S.A.S., Thermo Fisher Scientific Inc., and Sigma-Aldrich Co.). Melting points were measured on a MPA 100 OptiMelt $^{\circledast}$ apparatus and are uncorrected. Nuclear magnetic resonance (NMR) spectra were acquired at $400 \mathrm{MHz}$ for ${ }^{1} \mathrm{H} N M R$ and at $100 \mathrm{MHz}$ for ${ }^{13} \mathrm{C}$ NMR on a Varian $400-\mathrm{MR}$ spectrometer with tetramethylsilane (TMS) as internal standard, at room temperature (rt). Chemical shifts $(\delta)$ are expressed 
in ppm relative to TMS. Splitting patterns are designed: $s$, singlet; $d$, doublet; $d d$, doublet of doublet; $t$, triplet; m, multiplet; sym m, symmetric multiplet; br s, broaden singlet; br t, broaden triplet. Coupling constants (J) are reported in Hertz $(\mathrm{Hz})$. Thin layer chromatography $(\mathrm{TLC})$ was realized on Macherey Nagel silica gel plates with fluorescent indicator and were visualized under a UV-lamp at $254 \mathrm{~nm}$ and $365 \mathrm{~nm}$. Column chromatography was performed with a CombiFlash $R_{f}$ Companion (Teledyne-Isco System) using RediSep packed columns. IR spectra were recorded on a Varian 640-IR FT-IR Spectrometer. Elemental analyses (C, H, N) of new compounds were determined on a Thermo Electron apparatus by 'Pôle Chimie Moléculaire-Welience', Faculté de Sciences Mirande, Université de Bourgogne, Dijon, France. LC-MS was accomplished using an HPLC combined with a Surveyor MSQ (Thermo Electron) equipped with APCI source.

General procedure for the $\mathbf{N}$-Csp $\mathbf{C s}^{2}$ bond formation (copper-catalyzed coupling). To a suspension of pyrrolidin2-one (5a) or pterolactam (1a) (1 equiv.), copper(I) iodide (0.5 equiv.), cesium carbonate (2 equiv.) and the appropriate aryl halide (iodide or bromide) (1.0 equiv.) in anhydrous 1,4-dioxane under inert atmosphere, was added dropwise the coupling ligand, $N, N^{\prime}$-dimethylethylene diamine (DMEDA, 1-1.2 equiv.) via a syringe. The mixture was then stirred at $60{ }^{\circ} \mathrm{C}$ for 24 hours, turning blue very quickly. After cooling to rt, the mixture was filtered to remove insoluble salts and the filter cake was washed with dichloromethane. The filtrate was concentrated in vacuo and the residue was partitioned between distilled water and dichloromethane. The organic layer was dried over $\mathrm{MgSO}_{4}$ and evaporated to dryness. The crude product was purified by flash chromatography on prepacked SiOH columns (40-63 $\mu \mathrm{m}$ ) (eluent EtOAc/n-heptane, gradient 0/100 to 100/0) to afford pure $\mathrm{N}$-arylated compounds $5 \mathrm{c}$ or $1 \mathrm{c}-\mathrm{g}$.

5-(3,4,5-Trimethoxyphenylamino)-1-(3,5-dichlorophenyl)-pyrrolidin-2-one (5c). The general copper coupling procedure was followed using 5-(3,4,5-trimethoxyphenylamino)pyrrolidin-2-one (5a, $1.00 \mathrm{~g}, 3.7 \mathrm{mmol}, 1,3-$ dichloro-5-iodobenzene (1.02 g, $3.7 \mathrm{mmol})$, cesium carbonate $(2.44 \mathrm{~g}, 7.5 \mathrm{mmol})$, copper(I) iodide (0.36 g, 1.9 $\mathrm{mmol})$, dioxane $(30 \mathrm{~mL})$ and DMEDA $(0.38 \mathrm{~g}, 0.47 \mathrm{~mL}, 4.3 \mathrm{mmol})$. The crude product was purified by flash chromatography to afford amine $5 \mathrm{c}$ as a white solid in $72 \%$ yield ( $1.1 \mathrm{~g}$ ); $\mathrm{mp}$ (EtOAc/n-heptane) $181-182{ }^{\circ} \mathrm{C}$; IR (KBr): v 3329, 3068, 2956, 2886, 1691, 1610, 1545, 1498, 1435, 1389, 1255, 1201, 1128, 1075, 1010, 985, 839, $758 \mathrm{~cm}^{-1} ;{ }^{1} \mathrm{H}$ NMR $\left(\mathrm{CDCl}_{3}, 400 \mathrm{MHz}\right): \delta 2.08-2.20\left(\mathrm{~m}, 1 \mathrm{H}, \mathrm{CH}_{2} \mathrm{CH}_{2} \mathrm{CH}\right), 2.47-2.65\left(\mathrm{~m}, 2 \mathrm{H}, \mathrm{CH}_{2} \mathrm{CH}_{2} \mathrm{CH}\right), 2.69-2.81$ $\left(\mathrm{m}, 1 \mathrm{H}, \mathrm{CH}_{2} \mathrm{CH}_{2} \mathrm{CH}\right), 3.76\left(\mathrm{~s}, 3 \mathrm{H}, \mathrm{OCH}_{3}\right), 3.79(\mathrm{~s}, 6 \mathrm{H}, 2 \mathrm{OCH}), 3.86(\mathrm{~d}, J 9.4 \mathrm{~Hz}, 1 \mathrm{H}, \mathrm{NH}), 5.53-5.62(\mathrm{~m}, 1 \mathrm{H}$, $\mathrm{CH}_{2} \mathrm{CH}_{2} \mathrm{CH}$ ), $5.82(\mathrm{~s}, 2 \mathrm{H}, \mathrm{ArH}), 7.17(\mathrm{t}, J 1.9 \mathrm{~Hz}, 1 \mathrm{H}, \mathrm{ArH}), 7.46(\mathrm{~d}, J 1.9 \mathrm{~Hz}, 2 \mathrm{H}, \mathrm{ArH}) \mathrm{ppm} ;{ }^{13} \mathrm{C} \mathrm{NMR}(\mathrm{CDCl}, 100$ $\mathrm{MHz}): \delta 26.7\left(\mathrm{CH}_{2}\right), 30.2\left(\mathrm{CH}_{2}\right), 56.1\left(2 \mathrm{CH}_{3}\right), 61.0\left(\mathrm{CH}_{3}\right), 72.1(\mathrm{CH}), 92.8(2 \mathrm{CH}), 121.8(2 \mathrm{CH}), 126.0(\mathrm{CH}), 131.8$ (C), 135.2 (2C), 139.4 (C), 141.1 (C), 154.2 (2C), 173.6 (C) ppm; LC/MS (APCl $) \mathrm{m} / z 412.06$ (MH ) (calcd for $\left.\mathrm{C}_{19} \mathrm{H}_{20} \mathrm{Cl}_{2} \mathrm{~N}_{2} \mathrm{O}_{4}: 411.29 \mathrm{~g} / \mathrm{mol}\right)$.

5-Methoxy-1-(3,4-dimethoxyphenyl)pyrrolidin-2-one (1d). The general copper coupling procedure was followed using pterolactam (1a, $5.00 \mathrm{~g}, 43.5 \mathrm{mmol}), 4$-bromoveratrole $(9.43 \mathrm{~g}, 43.5 \mathrm{mmol})$, cesium carbonate (30.68 g, $94.2 \mathrm{mmol})$, copper(I) iodide $(4.10 \mathrm{~g}, 21.7 \mathrm{mmol})$, dioxane (100 mL) and DMEDA (3.83 g, $5.50 \mathrm{~mL}$, $43.47 \mathrm{mmol})$. The crude product was purified by flash chromatography to provide 5-methoxy-1-(3,4dimethoxyphenyl)-pyrrolidin-2-one (1d) as a yellow oil in 20\% yield (2.18 g); IR (KBr): v 1696, 1593, 1513, 1452, 1172, $1117 \mathrm{~cm}^{-1} ;{ }^{1} \mathrm{H}$ NMR $\left(\mathrm{CDCl}_{3}, 400 \mathrm{MHz}\right): \delta 1.99$ (bdd, J 9.5, 9.2 Hz, $\left.1 \mathrm{H}, \mathrm{CH}_{2} \mathrm{CH}_{2} \mathrm{CH}\right), 2.09-2.21(\mathrm{~m}, 1 \mathrm{H}$, $\mathrm{CH}_{2} \mathrm{CH}_{2} \mathrm{CH}$ ), 2.33 (ddd, J 17.6, 9.7, $2.2 \mathrm{~Hz}, 1 \mathrm{H}, \mathrm{CH}_{2} \mathrm{CH}_{2} \mathrm{CH}$ ), 2.59 (dt, J 17.4, $9.3 \mathrm{~Hz}, 1 \mathrm{H}, \mathrm{CH}_{2} \mathrm{CH}_{2} \mathrm{CH}$ ), $3.15(\mathrm{~s}, 3 \mathrm{H}$, $\left.\mathrm{CH}_{3}\right), 3.73\left(\mathrm{~s}, 3 \mathrm{H}, \mathrm{OCH}_{3}\right), 3.74\left(\mathrm{~s}, 3 \mathrm{H}, 2 \mathrm{OCH}\right.$ ) $, 5.13\left(\mathrm{dd}, J 6.1 \mathrm{~Hz}, 1 \mathrm{H}, \mathrm{CH}_{2} \mathrm{CH}_{2} \mathrm{CH}\right), 6.74(\mathrm{~d}, J 6.7 \mathrm{~Hz}, 2 \mathrm{H}, \mathrm{ArH}), 6.84$ (dd, J 6.1, $2.1 \mathrm{~Hz}, 1 \mathrm{H}, \mathrm{ArH}), 7.02(\mathrm{~d}, J 2.1 \mathrm{~Hz}, 1 \mathrm{H}, \mathrm{ArH}) \mathrm{ppm} ;{ }^{13} \mathrm{C} \mathrm{NMR}\left(\mathrm{CDCl}_{3}, 100 \mathrm{MHz}\right): \delta 24.4\left(\mathrm{CH}_{2}\right), 29.6\left(\mathrm{CH}_{2}\right)$, $53.8\left(\mathrm{CH}_{3}\right), 55.8\left(\mathrm{CH}_{3}\right), 55.9\left(\mathrm{CH}_{3}\right), 92.4(\mathrm{CH}), 108.1(\mathrm{CH}), 111.2(\mathrm{C}), 115.9(\mathrm{C}), 131.0(\mathrm{C}), 147.3(\mathrm{C}), 148.9(\mathrm{C})$, 174.4 (C) ppm; Anal. Calcd for $\mathrm{C}_{13} \mathrm{H}_{17} \mathrm{NO}_{4}$ : C, 62.14; $\mathrm{H}, 6.82 ; \mathrm{N}, 5.57$. Found: $\mathrm{C}, 62.32 ; \mathrm{H}, 6.91 ; \mathrm{N}, 5.83 \%$. 
5-Methoxy-1-(3,4,5-trimethoxyphenyl)pyrrolidin-2-one (1e). The general copper coupling procedure was followed using pterolactam (1a, $2.33 \mathrm{~g}, 20.2 \mathrm{mmol})$, 5-bromo-1,2,3-trimethoxy-benzene (5.00 g, $20.2 \mathrm{mmol})$, cesium carbonate $(13.19 \mathrm{~g}, 40.5 \mathrm{mmol})$, copper(l) iodide (1.93 g, $10.1 \mathrm{mmol})$, dioxane (25 $\mathrm{mL})$ and DMEDA $(1.78 \mathrm{~g}, 2.56 \mathrm{~mL}, 20.2 \mathrm{mmol})$. The crude product was purified by flash chromatography (EtOAc/n-heptane gradient $0 / 100$ to $100 / 0)$ to provide 5-methoxy-1-(3,4,5-trimethoxy-phenyl)pyrrolidin-2-one (1e) as a white solid in 54\% yield (3.06 g); $\mathrm{mp}$ (EtOAc/n-heptane) $92-93{ }^{\circ} \mathrm{C}$ (decomposition); IR (KBr): v 1708, 1585, 1503, 1453, 1126, $1075 \mathrm{~cm}^{-1} ;{ }^{1} \mathrm{H}$ NMR $\left(\mathrm{CDCl}_{3}, 400 \mathrm{MHz}\right.$ ): $\delta$ 2.11-2.19 (dddd, J 13.7, 9.4, 2.3, $1.0 \mathrm{~Hz}, 1 \mathrm{H}, \mathrm{CH}_{2} \mathrm{CH}_{2} \mathrm{CH}_{\text {), }}$ 2.25-2.36 (m, $1 \mathrm{H}, \mathrm{CH}_{2} \mathrm{CH}_{2} \mathrm{CH}$ ), 2.51 (ddd, J 17.4, 9.8, $2.5 \mathrm{~Hz}, 1 \mathrm{H}, \mathrm{CH}_{2} \mathrm{CH}_{2} \mathrm{CH}$ ), 2.76 (dt, J 17.4, 9.4 Hz, $1 \mathrm{H}$, $\mathrm{CH}_{2} \mathrm{CH}_{2} \mathrm{CH}$ ), $3.32\left(\mathrm{~s}, 3 \mathrm{H}, \mathrm{CH}_{3}\right), 3.84\left(\mathrm{~s}, 3 \mathrm{H}, \mathrm{OCH}_{3}\right), 3.86(\mathrm{~s}, 6 \mathrm{H}, 2 \mathrm{OCH}), 5.26\left(\mathrm{dd}, J 6.1,1.2 \mathrm{~Hz}, 1 \mathrm{H}, \mathrm{CH}_{2} \mathrm{CH}_{2} \mathrm{CH}_{3}\right.$ ), $6.76(\mathrm{~s}, 2 \mathrm{H}, \mathrm{ArH}) \mathrm{ppm} ;{ }^{13} \mathrm{C} \mathrm{NMR}\left(\mathrm{CDCl}_{3}, 100 \mathrm{MHz}\right): \delta 24.6\left(\mathrm{CH}_{2}\right), 29.8\left(\mathrm{CH}_{2}\right), 53.9\left(\mathrm{CH}_{3}\right), 56.2\left(2 \mathrm{CH}_{3}\right), 60.9\left(\mathrm{CH}_{3}\right)$, $92.6(\mathrm{CH}), 101.6(2 \mathrm{CH}), 133.7$ (C), $136.4(\mathrm{C}), 153.3(2 \mathrm{C}), 174.5$ (C) ppm; LC/MS (APCl $) \mathrm{m} / \mathrm{z} 282.11\left(\mathrm{MH}^{+}\right)(\mathrm{calcd}$ for $\left.\mathrm{C}_{14} \mathrm{H}_{19} \mathrm{NO}_{5}: 281.31 \mathrm{~g} / \mathrm{mol}\right)$.

5-Methoxy-1-(4-nitrophenyl)pyrrolidin-2-one (1f). The general copper coupling procedure was followed using pterolactam (1a, $2.31 \mathrm{~g}, 20.1 \mathrm{mmol})$, 1-iodo-4-nitrobenzene (5.02 g, $20.1 \mathrm{mmol})$, cesium carbonate (13.06 g, $40.1 \mathrm{mmol})$, copper(I) iodide (1.91 g, $10.0 \mathrm{mmol})$, dioxane $(50 \mathrm{~mL})$ and DMEDA (1.77 g, $2.56 \mathrm{~mL}, 20.1 \mathrm{mmol})$. The crude product was purified by flash chromatography to provide 5-methoxy-1-(4-nitrophenyl)-pyrrolidin-2one (1f) as a cream solid in 70\% yield (3.3 g); mp (EtOAc/ $n$-heptane) 85-86 ${ }^{\circ} \mathrm{C}$; IR (KBr): v 1707, 1594, 1498, 1192, 1164, $1113 \mathrm{~cm}^{-1} ;{ }^{1} \mathrm{H}$ NMR ( $\left.\mathrm{CDCl}_{3}, 400 \mathrm{MHz}\right): \delta 2.20-2.36\left(\mathrm{~m}, 2 \mathrm{H}, \mathrm{CH}_{2} \mathrm{CH}_{2} \mathrm{CH}\right), 2.56$ (ddd, J 17.7, 9.2, 2.9 Hz, $1 \mathrm{H}, \mathrm{CH}_{2} \mathrm{CH}_{2} \mathrm{CH}$ ), $2.76\left(\mathrm{dt}, J\right.$ 19.3, $9.6 \mathrm{~Hz}, 1 \mathrm{H}, \mathrm{CH}_{2} \mathrm{CH}_{2} \mathrm{CH}$ ), $3.37\left(\mathrm{~s}, 3 \mathrm{H}, \mathrm{OCH}_{3}\right), 5.42$ (dd, J 5.6, $1.3 \mathrm{~Hz}, 1 \mathrm{H}$,

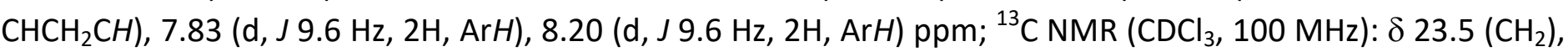
$30.1\left(\mathrm{CH}_{2}\right), 53.0\left(\mathrm{CH}_{3}\right), 90.9(\mathrm{CH}), 120.9(2 \mathrm{CH}), 124.4(2 \mathrm{CH}), 143.3(\mathrm{C}), 144.0(\mathrm{C}), 174.5(\mathrm{C})$. Anal. Calcd for $\mathrm{C}_{11} \mathrm{H}_{12} \mathrm{~N}_{2} \mathrm{O}_{4}$ : C, 55.93; $\mathrm{H}, 5.12 ; \mathrm{N}, 11.86$. Found: $\mathrm{C}, 56.27 ; \mathrm{H}, 5.29 ; \mathrm{N}, 12.09 \%$.

1-(1,1'-Biphenyl-4-yl)-5-methoxypyrrolidin-2-one (1g). The general copper coupling procedure was followed using pterolactam (1a, $1.65 \mathrm{~g}, 14.3 \mathrm{mmol})$, 4-iodobiphenyl (4.00 g, $14.3 \mathrm{mmol})$, cesium carbonate (9.31 g, 28.6 $\mathrm{mmol})$, copper(I) iodide $(1.36 \mathrm{~g}, 7.1 \mathrm{mmol})$, dioxane $(30 \mathrm{~mL})$ and $N, N^{\prime}$-dimethyl-ethylenediamine $(1.92 \mathrm{~mL}$, $14.34 \mathrm{mmol})$. The crude product was purified by flash chromatography to provide 5-methoxy-1-biphenyl-

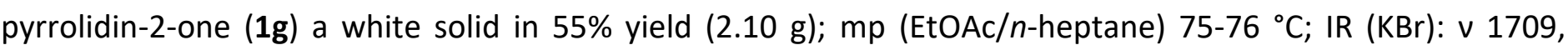
1609, 1520, 1485, 1456, 1192, 1166, $1129 \mathrm{~cm}^{-1} ;{ }^{1} \mathrm{H}$ NMR $\left(\mathrm{CDCl}_{3}, 400 \mathrm{MHz}\right): \delta 2.13$ (dddd, J 13.7, 9.3, $2.4,1.1$ $\left.\mathrm{Hz}, 1 \mathrm{H}, \mathrm{CH}_{2} \mathrm{CH}_{2} \mathrm{CH}\right), 2.20-2.30\left(\mathrm{~m}, 1 \mathrm{H}, \mathrm{CH}_{2} \mathrm{CH}_{2} \mathrm{CH}\right), 2.49\left(\mathrm{~m}, J 17.3,9.7,2.4 \mathrm{~Hz}, 1 \mathrm{H}, \mathrm{CH}_{2} \mathrm{CH}_{2} \mathrm{CH}\right), 2.76(\mathrm{dt}, J 17.4$, $8.7 \mathrm{~Hz}, 1 \mathrm{H}, \mathrm{CH}_{2} \mathrm{CH}_{2} \mathrm{CH}$ ), $3.29\left(\mathrm{~s}, 3 \mathrm{H}, \mathrm{OCH}_{3}\right.$ ), 5.35 (dd, J 6.0, 1.2 Hz, $1 \mathrm{H}, \mathrm{CH}_{2} \mathrm{CH}_{2} \mathrm{CH}$ ), 7.34 (ddt, J 8.1, 6.7, 1.3 Hz, $1 \mathrm{H}, \mathrm{ArH}), 7.42(\mathrm{bt}, J 7.6 \mathrm{~Hz}, 2 \mathrm{H}, \mathrm{ArH}), 7.56(\mathrm{bd}, J 7.3 \mathrm{~Hz}, 2 \mathrm{H}, \mathrm{ArH}), 7.60(\mathrm{~s}, 4 \mathrm{H}, \mathrm{ArH}) \mathrm{ppm} ;{ }^{13} \mathrm{C} \mathrm{NMR}\left(\mathrm{CDCl}_{3}, 100\right.$ $\mathrm{MHz}): \delta 24.3\left(\mathrm{CH}_{2}\right), 30.0\left(\mathrm{CH}_{2}\right), 53.5\left(\mathrm{CH}_{3}\right), 91.8(\mathrm{CH}), 123.2(2 \mathrm{CH}), 127.0(2 \mathrm{CH}), 127.3(\mathrm{CH}), 127.6(2 \mathrm{CH}), 128.8$ $(2 \mathrm{CH}), 137.2$ (C), 138.7 (C), 140.4 (C), 174.4 (C) ppm; Anal. Calcd for $\mathrm{C}_{17} \mathrm{H}_{17} \mathrm{NO}_{2}$ : C, 76.38; $\mathrm{H}, 6.41 ; \mathrm{N}, 5.24$. Found: C, 76.49; $\mathrm{H}, 6.66 ; \mathrm{N}, 5.17 \%$.

5-(3,4,5-Trimethoxyphenylamino)pyrrolidin-2-one (5a). ${ }^{15} \mathrm{~N}$-(Trimethylsilyl)-3,4,5-trimethoxyaniline (4a, 12.21 $\mathrm{g}, 47.8 \mathrm{mmol}$ ) was added to pterolactam (1a, $5.00 \mathrm{~g}, 43.4 \mathrm{mmol})$ in anhydrous dichloromethane $(40 \mathrm{~mL})$ and the solution was stirred under nitrogen atmosphere. TMSOTf $(0.25 \mathrm{~mL}, 0.307 \mathrm{~g}, 1.4 \mathrm{mmol})(5 \%$ vol.) was added through a syringe, and the resulting brownish, homogeneous solution was heated at $50{ }^{\circ} \mathrm{C}$ for 8 h. After cooling to room temperature, water $(100 \mathrm{~mL})$ and dichloromethane $(100 \mathrm{~mL})$ were added, and the mixture was intensively shaken in an extraction funnel, upon which the organic phase lost its brown color and remained slightly beige. The organic phase was washed with water $(3 \times 100 \mathrm{~mL})$ and dried $\left(\mathrm{MgSO}_{4}\right)$. The residue obtained upon evaporation was purified by flash chromatography (EtOAc/ $n$-heptane gradient $0 / 100$ to $100 / 0)$ to provide amine $5 \mathrm{a}$ as a white solid in $55 \%$ yield $(6.36 \mathrm{~g}) ; \mathrm{mp}(\mathrm{EtOH}) 181-182{ }^{\circ} \mathrm{C}$. IR $(\mathrm{KBr}): \mathrm{v} 3312,1685$, 1598, 1530, 1508, 1467, 1447, 1191, $1114 \mathrm{~cm}^{-1} ;{ }^{1} \mathrm{H} \mathrm{NMR}\left(\mathrm{CDCl}_{3}, 400 \mathrm{MHz}\right): \delta 1.85-1.96\left(\mathrm{~m}, 1 \mathrm{H}, \mathrm{CH}_{2} \mathrm{CH}_{2} \mathrm{CH}\right)$, 
2.30-2.51 (m, 2H, CH $\left.\mathrm{CH}_{2} \mathrm{CH}\right), 2.52-2.63\left(\mathrm{~m}, 1 \mathrm{H}, \mathrm{CH}_{2} \mathrm{CH}_{2} \mathrm{CH}\right), 3.77\left(\mathrm{~s}, 3 \mathrm{H}, \mathrm{OCH}_{3}\right), 3.82(\mathrm{~s}, 6 \mathrm{H}, 2 \mathrm{OCH}), 3.99(\mathrm{~d}, \mathrm{~J}$ $9.6 \mathrm{~Hz}, 1 \mathrm{H}, \mathrm{NH}), 5.20-5.27\left(\mathrm{~m}, 1 \mathrm{H}, \mathrm{CH}_{2} \mathrm{CH}_{2} \mathrm{CH}\right), 5.90(\mathrm{~s}, 2 \mathrm{H}, \mathrm{ArH}), 6.76(\mathrm{~s}, 1 \mathrm{H}, \mathrm{NH}) \mathrm{ppm} ;{ }^{13} \mathrm{C} \mathrm{NMR}\left(\mathrm{CDCl}_{3}, 100\right.$ $\mathrm{MHz}): \delta 28.9\left(\mathrm{CH}_{2}\right), 29.2\left(\mathrm{CH}_{2}\right), 56.0\left(2 \mathrm{CH}_{3}\right), 61.1\left(\mathrm{CH}_{3}\right), 65.5(\mathrm{CH}), 92.0(2 \mathrm{CH}), 131.5(\mathrm{C}), 142.1(\mathrm{C}), 154.2(2 \mathrm{C})$, 177.0 (C) ppm; LC/MS (APCl ${ }^{+}$m/z $267.16\left(\mathrm{MH}^{+}\right)$(calcd for $\mathrm{C}_{13} \mathrm{H}_{18} \mathrm{~N}_{2} \mathrm{O}_{4}: 266.29 \mathrm{~g} / \mathrm{mol}$ ).

Reaction of pterolactam 1a with $\mathbf{N}$-trimethylsilyl-3,4,5-trimethoxyaniline (4a). $\mathrm{N}$-(Trimethylsilyl)-3,4,5trimethoxyaniline $(4 \mathrm{a}, 12.21 \mathrm{~g}, 47.8 \mathrm{mmol})$ was added under nitrogen to pterolactam $(1 \mathrm{a}, 5.00 \mathrm{~g}, 43.4 \mathrm{mmol})$ in anhydrous dichloromethane $(40 \mathrm{~mL})$. Under stirring, TMSOTf $(0.5 \mathrm{~mL}, 0.704 \mathrm{~g}, 2.8 \mathrm{mmol})$ (10\% vol.) was added through a syringe, and the resulting brownish, homogeneous solution was heated at $50{ }^{\circ} \mathrm{C}$ for $14 \mathrm{~h}$. After cooling to room temperature, water $(100 \mathrm{~mL})$ and dichloromethane $(100 \mathrm{~mL})$ were added, and the mixture was intensively shaken in an extraction funnel, upon which the organic phase lost its brown color and remained slightly beige. The organic phase was washed with water $(3 \times 100 \mathrm{~mL})$ and dried $\left(\mathrm{MgSO}_{4}\right)$. The residue obtained upon evaporation was purified by flash chromatography (EtOAc/n-heptane gradient 0/100 to $100 / 0)$ to provide the following compounds.

5-(3,4,5-Trimethoxyphenylamino)pyrrolidin-2-one (5a). ${ }^{15}$ This product was obtained in $10 \%$ yield (1.15 g) as white crystals having a $\mathrm{mp}$ and spectral pattern identical to those of $\mathbf{5 a}$ obtained by the former above procedure.

3-(3-((3,4,5-Trimethoxyphenylcarbamoyl)-methyl)-5,6,7-trimethoxyquinol-in-2-yl)propanamide (7a). This product was obtained as white solid in $2 \%$ yield $(0.2 \mathrm{~g})$; $\mathrm{mp}$ (EtOAc/n-heptane) $201-203{ }^{\circ} \mathrm{C}$; IR (KBr): v 3374, 3259, 1662, 1601, 1538, 1509, 1486, 1450, $1126 \mathrm{~cm}^{-1} ;{ }^{1} \mathrm{H}$ NMR (DMSO-d $\left.6,400 \mathrm{MHz}\right): \delta 2.87(\mathrm{t}, J 7.4 \mathrm{~Hz}, 2 \mathrm{H}$, $\left.\mathrm{CH}_{2}\right), 3.19\left(\mathrm{t}, \mathrm{J} 7.4 \mathrm{~Hz}, 2 \mathrm{H}, \mathrm{CH}_{2}\right), 3.61\left(\mathrm{~s}, 3 \mathrm{H}, \mathrm{OCH}_{3}\right), 3.70\left(\mathrm{~s}, 2 \mathrm{H}, \mathrm{CH}_{2}\right), 3.72(\mathrm{~s}, 6 \mathrm{H}, 2 \mathrm{OCH}), 3.85(\mathrm{~s}, 3 \mathrm{H}, \mathrm{OCH})_{3}$, $3.91\left(\mathrm{~s}, 3 \mathrm{H}, \mathrm{OCH}_{3}\right), 3.97\left(\mathrm{~s}, 3 \mathrm{H}, \mathrm{OCH}_{3}\right), 7.02(\mathrm{~s}, 2 \mathrm{H}, \mathrm{ArH}), 7.03$ (bs, $\left.1 \mathrm{H}, \mathrm{NH}_{2}\right), 7.11(\mathrm{~s}, 1 \mathrm{H}, \mathrm{ArH}), 7.66(\mathrm{bs}, 1 \mathrm{H}, \mathrm{NH}$ ), $8.10(\mathrm{~s}, 1 \mathrm{H}, \mathrm{ArH}), 9.97$ (bs, $1 \mathrm{H}, \mathrm{NH}) \mathrm{ppm} ;{ }^{13} \mathrm{C}$ NMR (DMSO-d, $\left.100 \mathrm{MHz}\right): \delta 30.4\left(\mathrm{CH}_{2}\right), 34.7\left(\mathrm{CH}_{2}\right), 39.4\left(\mathrm{CH}_{2}\right)$, $56.1\left(2 \mathrm{CH}_{3}\right), 56.3\left(\mathrm{CH}_{3}\right), 60.5\left(\mathrm{CH}_{3}\right), 61.3\left(\mathrm{CH}_{3}\right), 61.9\left(\mathrm{CH}_{3}\right), 97.1(2 \mathrm{CH}), 103.9(\mathrm{CH}), 117.4(\mathrm{C}), 126.9(\mathrm{C}), 131.1$ (CH), 133.5 (C), 136.1 (C), 140.3 (C), 144.1 (C), 146.7 (C), 153.1 (2C), 155.5 (C), 159.8 (C), 171.4 (C), 172.3 (C) ppm; LC/MS $\left(\mathrm{APCl}^{+}\right) \mathrm{m} / \mathrm{z} 514.23\left(\mathrm{MH}^{+}\right.$) (calcd for $\mathrm{C}_{26} \mathrm{H}_{31} \mathrm{~N}_{3} \mathrm{O}_{8}: 513.55 \mathrm{~g} / \mathrm{mol}$ ).

3-(3-(Carbamoylmethyl)-5,6,7-trimethoxy-quinolin-2-yl)-N-(3,4,5-tri-methoxyphenyl)propanamide (8a). This product was obtained as white solid; $0.15 \%$ yield $(0.017 \mathrm{~g}) ; \mathrm{mp}$ (EtOAc/ $n$-heptane) $197-199{ }^{\circ} \mathrm{C}$ (decomposition); IR (KBr): v 3405, 3257, 1664, 1602, 1537, 1510, 1452, $1131 \mathrm{~cm}^{-1}$; ${ }^{1} \mathrm{H}$ NMR (DMSO-d $d_{6}, 400$ $\mathrm{MHz}$ ): $\delta 2.87\left(\mathrm{t}, J 7.5 \mathrm{~Hz}, 2 \mathrm{H}, \mathrm{CH}_{2}\right), 3.18\left(\mathrm{t}, J 7.5 \mathrm{~Hz}, 2 \mathrm{H}, \mathrm{CH}_{2}\right), 3.60\left(\mathrm{~s}, 3 \mathrm{H}, \mathrm{OCH}_{3}\right), 3.68\left(\mathrm{~s}, 2 \mathrm{H}, \mathrm{CH}_{2}\right), 3.72(\mathrm{~s}, 6 \mathrm{H}$, $\left.2 \mathrm{OCH}_{3}\right), 3.84\left(\mathrm{~s}, 3 \mathrm{H}, \mathrm{OCH}_{3}\right), 3.90\left(\mathrm{~s}, 3 \mathrm{H}, \mathrm{OCH}_{3}\right), 3.97\left(\mathrm{~s}, 3 \mathrm{H}, \mathrm{OCH}_{3}\right), 7.02\left(\mathrm{bs}, 3 \mathrm{H}, 2 \mathrm{ArH}+\mathrm{NH}_{2}\right), 7.11(\mathrm{~s}, 1 \mathrm{H}, \mathrm{ArH})$, 7.59 (bs, $1 \mathrm{H}, \mathrm{NH}_{2}$ ), 8.09 (s, $\left.1 \mathrm{H}, \mathrm{ArH}\right), 9.96$ (bs, $\left.1 \mathrm{H}, \mathrm{NH}\right) \mathrm{ppm} ;{ }^{13} \mathrm{C} \mathrm{NMR}\left(\mathrm{DMSO}-d_{6}, 100 \mathrm{MHz}\right): \delta 30.3\left(\mathrm{CH}_{2}\right), 34.7$ $\left(\mathrm{CH}_{2}\right), 39.3\left(\mathrm{CH}_{2}\right), 56.1\left(2 \mathrm{CH}_{3}\right), 56.2\left(\mathrm{CH}_{3}\right), 60.5\left(\mathrm{CH}_{3}\right), 61.3\left(\mathrm{CH}_{3}\right), 61.9\left(\mathrm{CH}_{3}\right), 97.1(2 \mathrm{CH}), 103.9(\mathrm{CH}), 117.4(\mathrm{C})$, 126.9 (CH), 131.0 (C), 131.8 (C), 136.1 (C), 140.3 (C), 144.1 (C), 146.7 (C), 153.1 (2C), 155.5 (C), 159.8 (C), 171.4 (C), 172.3 (C) ppm; LC/MS (APCl $\left.{ }^{+}\right) \mathrm{m} / z 514.23\left(\mathrm{MH}^{+}\right)$(calcd for $\mathrm{C}_{26} \mathrm{H}_{31} \mathrm{~N}_{3} \mathrm{O}_{8}: 513.55 \mathrm{~g} / \mathrm{mol}$ ).

3-(3-(Carbamoylmethylene)-5,6,7-trimethoxyquinolin-2-yl)propanamide (9a). This product was obtained as white solid; $1 \%$ yield $(0.076 \mathrm{~g}) ; \mathrm{mp}$ (EtOAc/n-heptane) $197-199{ }^{\circ} \mathrm{C} ; \mathrm{IR}(\mathrm{KBr}): \mathrm{v} 3414,3328,1661,1616,1571$, 1484, $1092 \mathrm{~cm}^{-1} ;{ }^{1} \mathrm{H}$ NMR (DMSO-d $6,400 \mathrm{MHz}$ ): $\delta 2.60$ (t, J $7.8 \mathrm{~Hz}, 2 \mathrm{H}, \mathrm{CH}_{2}$ ), 3.07 (t, J $7.8 \mathrm{~Hz}, 2 \mathrm{H}, \mathrm{CH}_{2}$ ), $3.66(\mathrm{~s}$, $\left.2 \mathrm{H}, \mathrm{CH}_{2}\right), 3.85\left(\mathrm{~s}, 3 \mathrm{H}, \mathrm{OCH}_{3}\right), 3.94\left(\mathrm{~s}, 3 \mathrm{H}, \mathrm{OCH}_{3}\right), 3.97\left(\mathrm{~s}, 3 \mathrm{H}, \mathrm{OCH}_{3}\right), 6.75\left(\mathrm{bs}, 1 \mathrm{H}, \mathrm{NH}_{2}\right), 7.00(\mathrm{bs}, 1 \mathrm{H}, \mathrm{NH}$ ), 7.13 $(\mathrm{s}, 1 \mathrm{H}, \mathrm{ArH}), 7.34$ (bs, $1 \mathrm{H}, \mathrm{NH}_{2}$ ), 7.56 (bs, $1 \mathrm{H}, \mathrm{NH}_{2}$ ), 8.07 (s, $\left.1 \mathrm{H}, \mathrm{ArH}\right) \mathrm{ppm} ;{ }^{13} \mathrm{C} \mathrm{NMR}$ (DMSO-d, $100 \mathrm{MHz}$ ): $\delta 30.6$ $\left(\mathrm{CH}_{2}\right), 33.5\left(\mathrm{CH}_{2}\right), 39.3\left(\mathrm{CH}_{2}\right), 56.3\left(\mathrm{CH}_{3}\right), 61.3\left(\mathrm{CH}_{3}\right), 61.9\left(\mathrm{CH}_{3}\right), 104.0(\mathrm{CH}), 117.4(\mathrm{C}), 126.9(\mathrm{C}), 131.0(\mathrm{CH})$, 140.2 (C), 144.2 (C), 146.6 (C), 155.5 (C), 160.2 (C), 172.4 (C), 174.5 (C) ppm; LC/MS (APCl $) \mathrm{m} / z 348.14\left(\mathrm{MH}^{+}\right)$ (calcd for $\mathrm{C}_{17} \mathrm{H}_{21} \mathrm{~N}_{3} \mathrm{O}_{5}: 347.37 \mathrm{~g} / \mathrm{mol}$ ).

4-(3,4,5-Trimethoxyphenylamino)butanamide (10a). This product was obtained in traces as slightly brown oil; ${ }^{1} \mathrm{H} \mathrm{NMR}\left(\mathrm{CDCl}_{3}, 400 \mathrm{MHz}\right): \delta 1.93\left(\mathrm{q}, J 6.9 \mathrm{~Hz} 2 \mathrm{H}, \mathrm{CH}_{2}\right), 2.33\left(\mathrm{t}, J 7.2 \mathrm{~Hz}, 2 \mathrm{H}, \mathrm{CH}_{2}\right), 3.12\left(\mathrm{t}, J 6.39 \mathrm{~Hz}, 2 \mathrm{H}, \mathrm{CH}_{2}\right)$, 
$3.74\left(\mathrm{~s}, 3 \mathrm{H}, \mathrm{OCH}_{3}\right), 3.80(\mathrm{~s}, 6 \mathrm{H}, 2 \mathrm{OCH}), 5.85(\mathrm{~s}, 2 \mathrm{H}, \mathrm{ArH}), 6.07(\mathrm{~s}, 1 \mathrm{H}, \mathrm{NH}), 6.21(\mathrm{~s}, 1 \mathrm{H}, \mathrm{NH}) \mathrm{ppm} ;{ }^{13} \mathrm{C} \mathrm{NMR}$ $\left(\mathrm{CDCl}_{3}, 100 \mathrm{MHz}\right): \delta 24.9\left(\mathrm{CH}_{2}\right), 33.0\left(\mathrm{CH}_{2}\right), 43.7\left(\mathrm{CH}_{2}\right), 55.9\left(2 \mathrm{CH}_{3}\right), 61.0\left(\mathrm{CH}_{3}\right), 90.2(2 \mathrm{CH}), 129.6(\mathrm{C}), 145.3(\mathrm{C})$, 153.8 (2C), 175.7 (C).

Reaction of $\mathbf{N}$-phenylpterolactam 1a with $\mathbf{N}$-(trimethylsilyl)anisidine (4b). $\mathbf{N}$-(Trimethylsilyl)anisidine (4b, $1.12 \mathrm{~g}, 5.7 \mathrm{mmol}$ ) was added under nitrogen to $\mathrm{N}$-phenyl pterolactam (1b, $1.00 \mathrm{~g}, 5.2 \mathrm{mmol}$ ) in anhydrous dichloromethane $(15 \mathrm{~mL})$. Under stirring, triflic acid $(0.20 \mathrm{~mL}, 0.339 \mathrm{~g}, 2.3 \mathrm{mmol})(20 \%$ vol.) was added through a syringe, and the resulting brownish, homogeneous solution was heated at $40{ }^{\circ} \mathrm{C}$ for $96 \mathrm{~h}$. After cooling to room temperature, water $(100 \mathrm{~mL})$ and dichloromethane $(100 \mathrm{~mL})$ were added, and the mixture was intensively shaken in an extraction funnel, upon which the organic phase lost its brown color and remained slightly beige. The organic phase was washed with water $(3 \times 100 \mathrm{~mL})$ and dried $\left(\mathrm{MgSO}_{4}\right)$. The residue obtained upon evaporation was purified by flash chromatography (EtOAc/n-heptane gradient $0 / 100$ to 100/0) to provide the following compounds.

3-(3-((Phenylcarbamoyl)methyl)-6-methoxyquinolin-2-yl)-N-phenylpropanamide (9b). This product was obtained as white solid; 30\% yield (0.345 g); mp (EtOAc/n-heptane) 202-204 ${ }^{\circ} \mathrm{C}$; IR (KBr): v 3299, 1656, 1599, 1533, 1493, 1235, $1029 \mathrm{~cm}^{-1}{ }^{1}{ }^{1} \mathrm{H}$ NMR (DMSO-d $\left.d_{6}, 400 \mathrm{MHz}\right): \delta 2.94\left(\mathrm{t}, J 7.5 \mathrm{~Hz}, 2 \mathrm{H}, \mathrm{CH}_{2}\right.$ ), $3.24(\mathrm{t}, J 7.5 \mathrm{~Hz}, 2 \mathrm{H}$, $\left.\mathrm{CH}_{2}\right), 3.87\left(\mathrm{~s}, 3 \mathrm{H}, \mathrm{OCH}_{3}\right), 3.98\left(\mathrm{~s}, 2 \mathrm{H}, \mathrm{CH}_{2}\right), 7.00(\mathrm{t}, J 7.4 \mathrm{~Hz}, 1 \mathrm{H}, \mathrm{ArH}), 7.05(\mathrm{t}, J 7.4 \mathrm{~Hz}, 1 \mathrm{H}, \operatorname{ArH}), 7.24-7.34(\mathrm{~m}$, $6 \mathrm{H}, \mathrm{ArH}), 7.61(\mathrm{t}, J 8.8 \mathrm{~Hz}, 4 \mathrm{H}, \mathrm{ArH}), 7.80(\mathrm{~d}, J 8.8 \mathrm{~Hz}, 1 \mathrm{H}, \mathrm{ArH}), 8.06(\mathrm{~s}, 1 \mathrm{H}, \mathrm{ArH}), 10.05(\mathrm{~s}, 1 \mathrm{H}, \mathrm{NH}), 10.31(\mathrm{~s}, 1 \mathrm{H}$, $\mathrm{NH}) \mathrm{ppm} ;{ }^{13} \mathrm{C}$ NMR (DMSO-d, $\left.100 \mathrm{MHz}\right): \delta 29.6\left(\mathrm{CH}_{2}\right), 34.0\left(\mathrm{CH}_{2}\right), 38.8\left(\mathrm{CH}_{2}\right), 55.4\left(\mathrm{CH}_{3}\right), 105.2(\mathrm{CH}), 118.9$ $(2 \mathrm{CH}), 119.2(2 \mathrm{CH}), 121.1(\mathrm{CH}), 122.7(\mathrm{CH}), 123.2(\mathrm{CH}), 127.5(\mathrm{CH}), 128.5(2 \mathrm{CH}), 128.6(2 \mathrm{CH}), 129.4(\mathrm{CH}), 129.9$ (C), 135.4 (CH), 139.0 (C), 139.4 (C), 141.9 (C), 156.8 (C), 157.3 (C), 168.5 (C), 171.0 (C) ppm; LC/MS (APCl $\left.{ }^{+}\right) \mathrm{m} / \mathrm{z}$ $440.21\left(\mathrm{MH}^{+}\right.$) (calcd for $\mathrm{C}_{27} \mathrm{H}_{25} \mathrm{~N}_{3} \mathrm{O}_{3}: 439.52 \mathrm{~g} / \mathrm{mol}$ ).

4-(4-Methoxyphenylamino)- $\mathbf{N}$-phenylbutanamide (10b). This product was obtained as brown solid; $5 \%$ yield $(0.075 \mathrm{~g}) ; \mathrm{mp}(\mathrm{EtOH}) 88-89^{\circ} \mathrm{C} ; \mathrm{IR}(\mathrm{KBr}): \mathrm{v} 3335,1650,1597,1512,1441,1233,1030 \mathrm{~cm}^{-1} ;{ }^{1} \mathrm{H} \mathrm{NMR}\left(\mathrm{CDCl}{ }_{3}, 400\right.$ $\mathrm{MHz}$ ): $\delta 1.95\left(\mathrm{q}, 2 \mathrm{H}, J 6.9 \mathrm{~Hz}, \mathrm{CH}_{2}\right), 2.41\left(\mathrm{t}, J 7.2 \mathrm{~Hz}, 2 \mathrm{H}, \mathrm{CH}_{2}\right), 3.09\left(\mathrm{t}, J 6.7 \mathrm{~Hz}, 2 \mathrm{H}, \mathrm{CH}_{2}\right), 3.51-3.62(\mathrm{~m}, 1 \mathrm{H}, \mathrm{NH})$, $3.71\left(\mathrm{~s}, 3 \mathrm{H}, \mathrm{OCH}_{3}\right), 6.54(\mathrm{dt}, J$ 8.9, 3.0 Hz, 2H, ArH), $6.75(\mathrm{dt}, J$ 8.9, 3.0 Hz, 2H, ArH), $7.06(\mathrm{t}, J 7.4 \mathrm{~Hz}, 1 \mathrm{H}, \mathrm{ArH})$, $7.26(\mathrm{t}, J 7.4 \mathrm{~Hz}, 2 \mathrm{H}, \mathrm{ArH}), 7.46(\mathrm{t}, J 7.9 \mathrm{~Hz}, 2 \mathrm{H}, \mathrm{ArH}), 7.92(\mathrm{~s}, 1 \mathrm{H}, \mathrm{NH}) \mathrm{ppm} ;{ }^{13} \mathrm{C} \mathrm{NMR}\left(\mathrm{CDCl}_{3}, 100 \mathrm{MHz}\right): \delta 25.2$ $\left(\mathrm{CH}_{2}\right), 35.0\left(\mathrm{CH}_{2}\right), 44.3\left(\mathrm{CH}_{2}\right), 55.8\left(\mathrm{CH}_{3}\right), 114.2(2 \mathrm{CH}), 114.9(2 \mathrm{CH}), 120.0(2 \mathrm{CH}), 124.2(\mathrm{CH}), 128.9(2 \mathrm{CH}), 138.0$ (C), 142.4 (2C), 152.1 (C), 171.3 (C) ppm; LC/MS ( $\left.\mathrm{APCl}^{+}\right) \mathrm{m} / z 285.14\left(\mathrm{MH}^{+}\right.$) (calcd for $\mathrm{C}_{17} \mathrm{H}_{20} \mathrm{~N}_{2} \mathrm{O}_{2}: 284.36 \mathrm{~g} / \mathrm{mol}$ ). 1,2-Bis(4-Methoxyphenyl)diazene (11b). This product was obtained as yellow solid with the same physicochemical properties as described in the literature; ${ }^{57} 1 \%$ yield $(0.006 \mathrm{~g}) ; \mathrm{mp}$ (EtOAc/n-heptane) 162-164 $\left.{ }^{\circ} \mathrm{C} ;{ }^{57} 155-159{ }^{\circ} \mathrm{C}\right) ;{ }^{1} \mathrm{H}$ NMR $\left.\left(\mathrm{CDCl}_{3}, 400 \mathrm{MHz}\right): \delta 3.89(\mathrm{~s}, 6 \mathrm{H}, 2 \mathrm{OCH})_{3}\right), 7.00(\mathrm{~d}, J 8.9 \mathrm{~Hz}, 2 \mathrm{H}, \mathrm{ArH}), 7.88(\mathrm{~d}, J 8.9 \mathrm{~Hz}$, $2 \mathrm{H}, \mathrm{ArH}) \mathrm{ppm}$.

Reaction of $\mathbf{N}$-3,5-dichlorophenylpterolactam 1c with $\mathbf{N}$-trimethylsilyl-3,4,5-trimethoxyaniline (4a). $\mathrm{N}$ Trimethylsilyl-3,4,5-trimethoxyaniline (4a, $1.08 \mathrm{~g}, 4.22 \mathrm{mmol}$ ) was added under nitrogen atmosphere to $\mathrm{N}$-3,5dichlorophenyl pterolactam (1c, $1.00 \mathrm{~g}, 3.84 \mathrm{mmol})$ in anhydrous dichloromethane $(15 \mathrm{~mL})$. Under stirring, triflic acid $(0.10 \mathrm{~mL}, 0.17 \mathrm{~g}, 1.12 \mathrm{mmol})(10 \%$ vol.) and glacial acetic acid $(0.10 \mathrm{~mL})(10 \%$ vol.) were added through a syringe, and the resulting brownish, homogeneous solution was heated at $40{ }^{\circ} \mathrm{C}$ for 96 hours. After cooling of the reaction to room temperature, water $(100 \mathrm{~mL})$ and dichloromethane $(100 \mathrm{~mL})$ were added, and the mixture was intensively shaken in an extraction funnel, upon which the organic phase lost its brown color and remained slightly beige. The organic phase was washed with water $(3 \times 100 \mathrm{~mL})$ and dried $\left(\mathrm{MgSO}_{4}\right)$. The residue obtained upon evaporation was purified by flash chromatography (EtOAc/ $n$-heptane gradient $0 / 100$ to $100 / 0)$ to provide the following compounds.

3-(3-((3,5-Dichlorophenylcarbamoyl)methyl)-5,6,7-trimethoxyquinolin-2-yl)-N-(3,5-dichlorophenyl)propanamide (9c). This product was obtained as white solid in $53 \%$ yield (0.65 g); mp (EtOAc/n-heptane) $257-259{ }^{\circ} \mathrm{C}$ 
(decomposition); IR (KBr): v 3292, 1680, 1583, 1520, 1443, $1099 \mathrm{~cm}^{-1} ;{ }^{1} \mathrm{H}$ NMR (DMSO- $\left.d_{6}, 400 \mathrm{MHz}\right): \delta 2.92(\mathrm{t}, J$ $\left.7.1 \mathrm{~Hz}, 2 \mathrm{H}, \mathrm{CH}_{2}\right), 3.22\left(\mathrm{t}, J 7.1 \mathrm{~Hz}, 2 \mathrm{H}, \mathrm{CH}_{2}\right), 3.85\left(\mathrm{~s}, 3 \mathrm{H}, \mathrm{OCH}_{3}\right), 3.91\left(\mathrm{~s}, 3 \mathrm{H}, \mathrm{OCH}_{3}\right), 3.98(\mathrm{~s}, 3 \mathrm{H}, \mathrm{OCH}), 4.01(\mathrm{~s}$, $\left.2 \mathrm{H}, \mathrm{CH}_{2}\right), 7.09(\mathrm{~s}, 1 \mathrm{H}, \mathrm{ArH}), 7.22(\mathrm{t}, J 1.8 \mathrm{~Hz}, 1 \mathrm{H}, \operatorname{ArH}), 7.26(\mathrm{t}, J 1.8 \mathrm{~Hz}, 1 \mathrm{H}, \operatorname{ArH}), 7.66(\mathrm{~d}, J 1.8 \mathrm{~Hz}, 2 \mathrm{H}, \operatorname{ArH}), 7.67$ (d, J $1.8 \mathrm{~Hz}, 2 \mathrm{H}, \mathrm{ArH}) 8.17(\mathrm{~s}, 1 \mathrm{H}, \mathrm{ArH}), 10.38(\mathrm{~s}, 1 \mathrm{H}, \mathrm{NH}), 10.62$ (s, 1H, NH) ppm; ${ }^{13} \mathrm{C}$ NMR (DMSO-d, $100 \mathrm{MHz}$ ): $\delta 30.0\left(\mathrm{CH}_{2}\right), 34.6\left(\mathrm{CH}_{2}\right), 40.4\left(\mathrm{CH}_{2}\right), 56.2\left(\mathrm{CH}_{3}\right), 61.3\left(\mathrm{CH}_{3}\right), 61.9\left(\mathrm{CH}_{3}\right), 103.8(\mathrm{CH}), 117.3(\mathrm{C}), 117.4(2 \mathrm{CH}), 117.8$ $(2 \mathrm{CH}), 122.4(\mathrm{CH}), 123.0(\mathrm{CH}), 125.8(\mathrm{C}), 131.7(\mathrm{CH}), 134.5(2 \mathrm{C}), 134.6(2 \mathrm{C}), 140.4(\mathrm{C}), 141.8(\mathrm{C}), 142.2(\mathrm{C}), 144.2$ (C), 146.7 (C), 155.7 (C), 159.5 (C), 170.1 (C), 172.4 (C) ppm; LC/MS (APCl ) m/z 638.05 (MH ${ }^{+}$) (calcd for $\left.\mathrm{C}_{29} \mathrm{H}_{25} \mathrm{Cl}_{4} \mathrm{~N}_{3} \mathrm{O}_{5}: 637.35 \mathrm{~g} / \mathrm{mol}\right)$.

4-(3,4,5-Trimethoxyphenylamino)- $\mathbf{N}$-(3,5-dichlorophenylbutanamide (10c). This product was obtained as brown oil in traces; ${ }^{1} \mathrm{H}$ NMR $\left(\mathrm{CDCl}_{3}, 400 \mathrm{MHz}\right): \delta 1.97-2.06\left(\mathrm{~m}, 2 \mathrm{H}, \mathrm{CH}_{2}\right), 2.47\left(\mathrm{t}, J 7.0 \mathrm{~Hz}, 2 \mathrm{H}, \mathrm{CH}_{2}\right), 3.17(\mathrm{t}, J 7.0$ $\left.\mathrm{Hz}, 2 \mathrm{H}, \mathrm{CH}_{2}\right), 3.76\left(\mathrm{~s}, 3 \mathrm{H}, \mathrm{OCH}_{3}\right), 3.77(\mathrm{~s}, 1 \mathrm{H}, \mathrm{NH}), 3.79(\mathrm{~s}, 6 \mathrm{H}, 2 \mathrm{OCH})_{3}, 5.84(\mathrm{~s}, 2 \mathrm{H}, \mathrm{ArH}), 7.07(\mathrm{t}, J 2.0 \mathrm{~Hz}, 1 \mathrm{H}$,

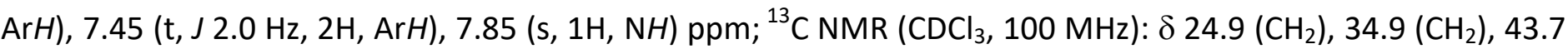
$\left(\mathrm{CH}_{2}\right), 55.9\left(2 \mathrm{CH}_{3}\right), 61.1\left(\mathrm{CH}_{3}\right), 90.5(2 \mathrm{CH}), 117.9(2 \mathrm{CH}), 124.0(\mathrm{CH}), 135.1(2 \mathrm{C}), 139.8(\mathrm{C}), 143.0(\mathrm{C}), 145.4(\mathrm{C})$, $154.0(2 \mathrm{C}), 171.3(\mathrm{C})$.

(E)-Bis(3,4,5-Trimethoxyphenyl)diazene (11c). This product was obtained as yellow-orange solid; $18 \%$ yield (0.138 g); mp (EtOAc/n-heptane) $208-209{ }^{\circ} \mathrm{C} ;{ }^{58} 217-218{ }^{\circ} \mathrm{C}$ ) with the same physicochemical characteristics as previously described in reference. ${ }^{58}$

\section{General procedures of reaction of $\boldsymbol{N}$-aryl pterolactam 1 with TMSOTf}

Method A. TMSOTf ( $0.05 \mathrm{~mL}$ for $1 \mathrm{~g}$ of lactam, $5 \%$ vol.) was added through a syringe to a stirred solution of $\mathrm{N}$ aryl pterolactam 1 in $\mathrm{CH}_{2} \mathrm{Cl}_{2}(5 \mathrm{~mL})$ placed under nitrogen. The brownish homogeneous solution was stirred at $40{ }^{\circ} \mathrm{C}$ for 50-100 hours. After cooling to room temperature, water $(25 \mathrm{~mL})$ and dichloromethane $(25 \mathrm{~mL})$ were added, and the mixture was intensively shaken in an extraction funnel, upon which the organic phase lost its brown color and remained slightly beige. The organic phase was washed repeatedly with water $(3 \times 25 \mathrm{~mL})$ and dried $\left(\mathrm{MgSO}_{4}\right)$. The residue obtained upon evaporation was purified by flash chromatography on prepacked SiOH column (40-63 $\mu \mathrm{m}$ ) (eluent: EtOAc/n-heptane, gradient 0/100 to 100/0).

Method B. TMSOTf ( $0.5 \mathrm{~mL}$ for $1 \mathrm{~g}$ of lactam, $50 \%$ vol.) was added through a syringe to a stirred solution of $N$ aryl pterolactam 1 in $\mathrm{CH}_{2} \mathrm{Cl}_{2}(5 \mathrm{~mL})$ placed under nitrogen. The brownish, homogeneous solution was stirred at rt for 1-2 h. Water $(25 \mathrm{~mL})$ and dichloromethane $(25 \mathrm{~mL})$ were added, and the mixture was intensively shaken in an extraction funnel, upon which the organic phase lost its brown color and remained slightly beige. The organic phase was washed repeatedly with water $(3 \times 25 \mathrm{~mL})$ and dried $\left(\mathrm{MgSO}_{4}\right)$. The residue obtained upon evaporation was purified by flash chromatography on prepacked SiOH column (eluent: EtOAc/n-heptane, gradient $0 / 100$ to $100 / 0)$.

Reaction of N-(3,5-dichlorophenyl) pterolactam 1c with TMSOTf. The following compounds were obtained using Method A and Method B.

Method A. N-(3,5-Dichlorophenyl)pterolactam (1c, $1.0 \mathrm{~g}, 3.85 \mathrm{mmol})$ and TMSOTf $(0.05 \mathrm{~mL}, 0.04 \mathrm{~g}, 0.18$ $\mathrm{mmol}$ ) in $\mathrm{CH}_{2} \mathrm{Cl}_{2}$ were stirred at $40{ }^{\circ} \mathrm{C}$ for $72 \mathrm{~h}$. Method B. $\mathrm{N}$-(3,5-Dichlorophenyl)pterolactam (1c, $0.50 \mathrm{~g}, 1.92$ $\mathrm{mmol})$ and TMSOTf $(0.25 \mathrm{~mL}, 0.204 \mathrm{~g}, 0.91 \mathrm{mmol})$ in $15 \mathrm{~mL}$ of $\mathrm{CH}_{2} \mathrm{Cl}_{2}$ were stirred at rt for $1.5 \mathrm{~h}$.

\section{9,11-Dichloro-1-(3,5-dichlorophenyl)-3a,4,5,11b-tetrahydro-1H-dipyrrolo[1,2-a:3',2'-c]quinoline-}

2,6(3H,3bH)-dione (14c). This product was obtained as white solid (Method A. 30\% yield (0.263 g); Method B. $5 \%$ yield (0.022g)); mp (EtOAc/n-heptane) 197-200 ${ }^{\circ} \mathrm{C}$; IR (KBr): v 1698, 1583, 1565, 1441, 1154, $1130 \mathrm{~cm}^{-1} ;{ }^{1} \mathrm{H}$ NMR (DMSO-d $d_{6}, 400 \mathrm{MHz}$ ): $\delta 1.74$ (quint, J $10.4 \mathrm{~Hz}, 1 \mathrm{H}, \mathrm{CH}_{2} \mathrm{CH}_{2} \mathrm{CH}$ ), $2.33\left(\mathrm{~d}, J 16.5 \mathrm{~Hz}, 1 \mathrm{H}, \mathrm{CH}_{2} \mathrm{CH}_{2} \mathrm{CH}\right.$ ), 2.53$2.58\left(\mathrm{~m}, 2 \mathrm{H}, \mathrm{CH}_{2} \mathrm{CH}_{2} \mathrm{CH}\right.$ ), 2.61-2.73 (m, 2H, $\mathrm{CH}_{2} \mathrm{CH}_{2} \mathrm{CH}$ ), 3.02 (dd, J 16.5, $6.5 \mathrm{~Hz}, 1 \mathrm{H}, \mathrm{CH}_{2} \mathrm{CH}_{2} \mathrm{CH}$ ), 3.96-4.06 (sym m, J $6.7 \mathrm{~Hz}, 1 \mathrm{H}, \mathrm{CH}), 5.36(\mathrm{~d}, J 4.3 \mathrm{~Hz}, 1 \mathrm{H}, \mathrm{CH}), 6.95(\mathrm{~d}, J 2.1 \mathrm{~Hz}, 2 \mathrm{H}, \operatorname{ArH}), 7.22(\mathrm{~d}, J 1.9 \mathrm{~Hz}, 1 \mathrm{H}, \operatorname{ArH}), 7.52(\mathrm{t}, J$ 
$1.9 \mathrm{~Hz}, 1 \mathrm{H}, \mathrm{ArH}$ ), $8.92(\mathrm{~d}, J 2.1 \mathrm{~Hz}, 1 \mathrm{H}, \mathrm{ArH}) \mathrm{ppm} ;{ }^{13} \mathrm{C}$ NMR (DMSO-d, $100 \mathrm{MHz}$ ): $\delta 22.5\left(\mathrm{CH}_{2}\right), 31.5\left(\mathrm{CH}_{2}\right), 33.5$ $\left(\mathrm{CH}_{2}\right), 36.0(\mathrm{CH}), 54.7(\mathrm{CH}), 57.6(\mathrm{CH}), 116.5(2 \mathrm{CH}), 117.5(\mathrm{CH}), 122.7(\mathrm{C}), 126.9(\mathrm{CH}), 127.9(2 \mathrm{C}), 133.1(\mathrm{CH})$, 134.4 (C), 137.1 (C), 139.3 (C), 139.7 (C), 172.5 (C), 174.5 (C) ppm; Anal. Calcd for $\mathrm{C}_{20} \mathrm{H}_{14} \mathrm{Cl}_{4} \mathrm{~N}_{2} \mathrm{O}_{2}: \mathrm{C}, 52.66 ; \mathrm{H}$, $3.09 ; \mathrm{N}, 6.14$. Found: $\mathrm{C}, 52.98 ; \mathrm{H}, 3.41 ; \mathrm{N}, 5.88 \%$.

5,5'-(1-(3,5-dichlorophenyl)-2-oxo-2,3-dihydro-1H-pyrrole-3,4-diyl)bis(1-(3,5-dichlorophenyl)pyrrolidin-2one) (16c). Following the Method B, this product was obtained as white solid in $90 \%$ yield (0.395 g); $\mathrm{mp}(\mathrm{EtOH})$ 218-220 ${ }^{\circ} \mathrm{C}$; IR (KBr): v 1703, 1600, 1580, 1445, 1168, $1123 \mathrm{~cm}^{-1} ;{ }^{1} \mathrm{H}$ NMR (DMSO-d $\left.6,400 \mathrm{MHz}\right): \delta 0.88-0.95$ (m, $\left.1 \mathrm{H}, \mathrm{CH}_{2} \mathrm{CH}_{2} \mathrm{CH}\right), 1.46-1.58\left(\mathrm{~m}, 1 \mathrm{H}, \mathrm{CH}_{2} \mathrm{CH}_{2} \mathrm{CH}\right), 1.70-1.84\left(\mathrm{~m}, 1 \mathrm{H}, \mathrm{CH}_{2} \mathrm{CH}_{2} \mathrm{CH}\right), 1.88-2.06\left(\mathrm{~m}, 2 \mathrm{H}, \mathrm{CH}_{2} \mathrm{CH}_{2} \mathrm{CH}\right)$, 2.42-2.58 (m, 5H, $\left.\mathrm{CH}_{2} \mathrm{CH}_{2} \mathrm{CH}\right), 2.60-2.71\left(\mathrm{~m}, 1 \mathrm{H}, \mathrm{CH}_{2} \mathrm{CH}_{2} \mathrm{CH}\right), 4.56-4.62(\mathrm{~m}, 1 \mathrm{H}, \mathrm{CH}), 5.08(\mathrm{dd}, J 3.6,1.8 \mathrm{~Hz}, 1 \mathrm{H}$, $\mathrm{CH}), 5.33(\mathrm{~d}, J 8.0 \mathrm{~Hz}, 1 \mathrm{H}, \mathrm{CH}), 7.33(\mathrm{t}, J 1.8 \mathrm{~Hz}, 1 \mathrm{H}, \mathrm{ArH}), 7.43(\mathrm{t}, J 1.8 \mathrm{~Hz}, 1 \mathrm{H}, \mathrm{ArH}), 7.45(\mathrm{~s}, 1 \mathrm{H}, \mathrm{ArH}), 7.46(\mathrm{~d}, J$ $1.8 \mathrm{~Hz}, 1 \mathrm{H}, \mathrm{ArH}), 7.56(\mathrm{~d}, J 1.8 \mathrm{~Hz}, 1 \mathrm{H}, \mathrm{ArH}), 7.68(\mathrm{~d}, J 1.8 \mathrm{~Hz}, 1 \mathrm{H}, \mathrm{ArH}) \mathrm{ppm} ;{ }^{13} \mathrm{C} N M R\left(\mathrm{DMSO}-d_{6}, 100 \mathrm{MHz}\right): \delta$ $16.7\left(\mathrm{CH}_{2}\right), 25.0\left(\mathrm{CH}_{2}\right), 30.2\left(\mathrm{CH}_{2}\right), 31.3\left(2 \mathrm{CH}_{2}\right), 39.6(\mathrm{CH}), 54.9(\mathrm{CH}), 57.9(\mathrm{CH}), 61.5(\mathrm{CH}), 119.8(\mathrm{CH}), 119.9(\mathrm{C})$, $121.4(\mathrm{CH}), 122.8(2 \mathrm{CH}), 122.9(\mathrm{C}), 124.1(\mathrm{CH}), 125.3(\mathrm{CH}), 125.8(\mathrm{CH}), 134.6(\mathrm{C}), 134.8(\mathrm{C}), 135.0(\mathrm{C}), 138.9(\mathrm{C})$, 139.8 (C), 140.2 (C), 140.9 (C), 141.1 (2C), 168.4 (C), 173.5 (C), 174.7 (C) ppm; Anal. Calcd for $\mathrm{C}_{30} \mathrm{H}_{21} \mathrm{Cl}_{6} \mathrm{~N}_{3} \mathrm{O}_{3}: \mathrm{C}_{\text {, }}$ 52.66; H, 3.09; N, 6.14. Found: C, 52.78; H, 3.33; N, 6.37\%.

Reaction of $\mathbf{N}$-(3,4-dimethoxyphenyl)pterolactam 1d with TMSOTf. The following compounds were obtained following the use of Method A and Method B.

Method A: N-(3,5-Dimethoxyphenyl)pterolactam (1d, $0.7 \mathrm{~g}, 2.8 \mathrm{mmol})$ and TMSOTf $(0.04 \mathrm{~mL}, 0.043 \mathrm{~g}, 0.19$ $\mathrm{mmol}$ ) in $\mathrm{CH}_{2} \mathrm{Cl}_{2}$ were stirred at $40{ }^{\circ} \mathrm{C}$ for $50 \mathrm{~h}$. Method B: $\mathrm{N}$-(3,5-Dimethoxyphenyl)pterolactam (1d, $0.7 \mathrm{~g}, 2.8$ mmol) and TMSOTf (0.35 mL, $0.43 \mathrm{~g}, 1.9 \mathrm{mmol}$ ) in $15 \mathrm{~mL}$ of $\mathrm{CH}_{2} \mathrm{Cl}_{2}$ were stirred at rt for $1.5 \mathrm{~h}$.

\section{1-(3,4-Dimethoxyphenyl)-9,10-dimethoxy-3a,4,5,11b-tetrahydro-1H-dipyrrolo[1,2-a:3',2'-c]-quinoline-}

2,6(3H,3bH)-dione $(\mathbf{1 4 d}-\alpha, \beta)$. This product was obtained as white solid (Method A: $42 \%$ yield $(0.256 \mathrm{~g})$; Method B: $52 \%$ yield (0.318 g)); mp (EtOAc/n-heptane) 102-105 ${ }^{\circ} \mathrm{C}$; IR (KBr): v 1694, 1666, 1591, 1511, 1455, 1153, $1133 \mathrm{~cm}^{-1} ;{ }^{1} \mathrm{H}$ NMR $\left(\mathrm{CDCl}_{3}, 400 \mathrm{MHz}\right): \delta 1.72-1.89\left(\mathrm{~m}, 1 \mathrm{H}, \mathrm{CH}_{2} \mathrm{CH}_{2} \mathrm{CH}\right), 2.46\left(\mathrm{~d}, J 17.2 \mathrm{~Hz}, 1 \mathrm{H}, \mathrm{CH}_{2} \mathrm{CH}_{2} \mathrm{CH}\right)$, 2.45-2.77 (m, 4H, $\mathrm{CH}_{2} \mathrm{CH}_{2} \mathrm{CH}$ ), 2.98 (dd, J 17.2, 7.2 Hz, 1H, $\mathrm{CH}_{2} \mathrm{CH}_{2} \mathrm{CH}$ ), 3.35 (s, 3H, OCH $), 3.75(\mathrm{~s}, 3 \mathrm{H}, \mathrm{OCH})_{3}$, $3.86\left(\mathrm{~s}, 3 \mathrm{H}, \mathrm{OCH}_{3}\right), 3.84-3.91(\mathrm{~m}, 1 \mathrm{H}, \mathrm{CH}), 3.90\left(\mathrm{~s}, 3 \mathrm{H}, \mathrm{OCH}_{3}\right), 4.88(\mathrm{~d}, J 5.2 \mathrm{~Hz}, 1 \mathrm{H}, \mathrm{CH}), 5.90(\mathrm{~s}, 1 \mathrm{H}, \operatorname{ArH}), 6.46$ (d, J $2.2 \mathrm{~Hz}, 1 \mathrm{H}, \mathrm{ArH}), 6.49$ (dd, J 8.4, $2.2 \mathrm{~Hz}, 1 \mathrm{H}, \mathrm{ArH}), 6.83(\mathrm{~d}, J 8.4 \mathrm{~Hz}, 1 \mathrm{H}, \mathrm{ArH}), 8.38(\mathrm{~s}, 1 \mathrm{H}, \mathrm{ArH}) \mathrm{ppm} ;{ }^{13} \mathrm{C}$ NMR $\left(\mathrm{CDCl}_{3}, 100 \mathrm{MHz}\right): \delta 23.1\left(\mathrm{CH}_{2}\right), 31.6\left(\mathrm{CH}_{2}\right), 34.2\left(\mathrm{CH}_{2}\right), 38.3(\mathrm{CH}), 55.5\left(\mathrm{CH}_{3}\right), 55.9\left(\mathrm{CH}_{3}\right), 56.0(\mathrm{CH}, \mathrm{CH})$, $56.1\left(\mathrm{CH}_{3}\right), 60.6(\mathrm{CH}), 102.5(\mathrm{CH}), 111.4(\mathrm{CH}), 112.1(\mathrm{CH}), 112.9(\mathrm{CH}), 113.8(\mathrm{CH}), 121.6(\mathrm{C}), 129.4(\mathrm{C}), 130.4(\mathrm{C})$, 144.2 (C), 149.0 (C), 149.2 (C), 149.6 (C), 173.0 (C), 173.4 (C) ppm; Anal. Calcd for $\mathrm{C}_{24} \mathrm{H}_{26} \mathrm{~N}_{2} \mathrm{O}_{6}$ : C, 65.74; $\mathrm{H}, 5.98$; $\mathrm{N}, 6.39$. Found: $\mathrm{C}, 65.86 ; \mathrm{H}, 6.24 ; \mathrm{N}, 6.70 \%$.

1-(3,4-Dimethoxyphenyl)-5-hydroxypyrrolidin-2-one (19d). This product was obtained as yellow oil (Method A: $40 \%$ yield (0.264 g); Method B: Traces); IR (KBr): v 3347, 1668, 1594, 1512, 1452, 1199, $1168,1109 \mathrm{~cm}^{-1} ;{ }^{1} \mathrm{H}$ NMR $\left(\mathrm{CDCl}_{3}, 400 \mathrm{MHz}\right): \delta 1.90-1.97\left(\mathrm{~m}, 1 \mathrm{H}, \mathrm{CH}_{2} \mathrm{CH}_{2} \mathrm{CH}\right), 2.27-2.28\left(\mathrm{~m}, 2 \mathrm{H}, \mathrm{CH}_{2} \mathrm{CH}_{2} \mathrm{CH}\right), 2.59-2.69(\mathrm{~m}, 1 \mathrm{H}$, $\mathrm{CH}_{2} \mathrm{CH}_{2} \mathrm{CH}$ ), $3.79\left(\mathrm{~s}, 3 \mathrm{H}, \mathrm{OCH}_{3}\right), 3.81\left(\mathrm{~s}, 3 \mathrm{H}, \mathrm{OCH}_{3}\right), 4.45(\mathrm{~d}, J 7.1 \mathrm{~Hz}, 1 \mathrm{H}, \mathrm{OH}), 5.45(\mathrm{td}, J 6.4,2.0 \mathrm{~Hz}, 1 \mathrm{H}$, $\left.\mathrm{CH}_{2} \mathrm{CH}_{2} \mathrm{CH}\right), 6.78(\mathrm{~d}, J 8.6 \mathrm{~Hz}, 1 \mathrm{H}, \mathrm{ArH}), 6.90(\mathrm{dd}, J 8.6,2.4 \mathrm{~Hz}, 1 \mathrm{H}, \mathrm{ArH}), 7.04(\mathrm{~d}, J 2.4 \mathrm{~Hz}, 1 \mathrm{H}, \mathrm{ArH}) \mathrm{ppm} ;{ }^{13} \mathrm{CNMR}$ $\left(\mathrm{CDCl}_{3}, 100 \mathrm{MHz}\right): \delta 28.1\left(\mathrm{CH}_{2}\right), 29.5\left(\mathrm{CH}_{2}\right), 55.9\left(\mathrm{CH}_{3}\right), 56.0\left(\mathrm{CH}_{3}\right), 85.6(\mathrm{CH}), 108.4(\mathrm{CH}), 111.2(\mathrm{CH}), 116.3(\mathrm{CH})$, 130.3 (C), 147.4 (C), 149.0 (C), 174.7 (C) ppm; Anal. Calcd for $\mathrm{C}_{12} \mathrm{H}_{15} \mathrm{NO}_{4}$ : C, 60.75; H, 6.37; N, 5.90. Found: C, $61.01 ; \mathrm{H}, 6.49 ; \mathrm{N}, 6.22 \%$.

Reaction of $\mathbf{N}$-(3,4,5-trimethoxyphenyl)pterolactam 1 e with TMSOTf. The following compounds were obtained using Method A and Method B.

Method A. N-(3,4,5-Trimethoxyphenyl)pterolactam (1e, $1.00 \mathrm{~g}, 3.55 \mathrm{mmol})$ and TMSOTf $(0.05 \mathrm{~mL}, 0.061 \mathrm{~g}$, $0.27 \mathrm{mmol}$ ) in $\mathrm{CH}_{2} \mathrm{Cl}_{2}$ were stirred at $40{ }^{\circ} \mathrm{C}$ for $100 \mathrm{~h}$. Method B. $\mathrm{N}$-(3,4,5-Trimethoxyphenyl)pterolactam (1e, $1.0 \mathrm{~g}, 3.55 \mathrm{mmol})$ and TMSOTf $(0.5 \mathrm{~mL}, 0.61 \mathrm{~g}, 2.71 \mathrm{mmol})$ in $15 \mathrm{~mL}$ of $\mathrm{CH}_{2} \mathrm{Cl}_{2}$ were stirred at rt for $1.5 \mathrm{~h}$. 
9,10,11-trimethoxy-1-(3,4,5-trimethoxy-phenyl)-3a,4,5,11b-tetrahydro-1H-dipyrrolo[1,2-a:3',2'-c]quinoline2,6(3H,3bH)-dione (14e). This product was obtained as white solid (Method A: 35\% yield (0.310 g); Method B: $60 \%$ yield (0.532 g)); mp (EtOAc/n-heptane) $225-227^{\circ} \mathrm{C}$; IR (KBr): v 1687, 1591, 1492, 1454, 1132, $1114 \mathrm{~cm}^{-1}$; ${ }^{1} \mathrm{H}$ NMR (DMSO-d ${ }_{6}, 400 \mathrm{MHz}$ ): $\delta 1.69$ (q, J $10.4 \mathrm{~Hz}, 1 \mathrm{H}, \mathrm{CH}_{2} \mathrm{CH}_{2} \mathrm{CH}$ ), $2.23\left(\mathrm{~d}, J 16.4 \mathrm{~Hz}, 1 \mathrm{H}, \mathrm{CH}_{2} \mathrm{CH}_{2} \mathrm{CH}\right.$ ), $2.35-2.46$ (m, $3 \mathrm{H}, \mathrm{CH}_{2} \mathrm{CH}_{2} \mathrm{CH}$ ), 2.58-2.70 (m, $1 \mathrm{H}, \mathrm{CH}_{2} \mathrm{CH}_{2} \mathrm{CH}$ ), 2.92 (dd, J 16.4, 6.6 Hz, $1 \mathrm{H}, \mathrm{CH}_{2} \mathrm{CH}_{2} \mathrm{CH}$ ), $3.26(\mathrm{~s}, 3 \mathrm{H}, \mathrm{OCH}$ ), $3.43\left(\mathrm{~s}, 3 \mathrm{H}, \mathrm{OCH}_{3}\right), 3.54\left(\mathrm{~s}, 6 \mathrm{H}, 2 \mathrm{OCH} \mathrm{CH}_{3}\right), 3.58\left(\mathrm{~s}, 3 \mathrm{H}, \mathrm{OCH}_{3}\right), 3.77\left(\mathrm{~s}, 3 \mathrm{H}, \mathrm{OCH}_{3}\right), 3.82-3.91(\mathrm{~m}, 1 \mathrm{H}, \mathrm{CH}), 5.11(\mathrm{~d}, J$ $4.4 \mathrm{~Hz}, 1 \mathrm{H}, \mathrm{CH}), 6.04(\mathrm{~s}, 2 \mathrm{H}, \mathrm{ArH}), 8.27(\mathrm{~s}, 1 \mathrm{H}, \mathrm{ArH}) \mathrm{ppm} ;{ }^{13} \mathrm{C}$ NMR (DMSO-d, $\left.400 \mathrm{MHz}\right): \delta 23.2\left(\mathrm{CH}_{2}\right), 32.2$ $\left(\mathrm{CH}_{2}\right), 34.5\left(\mathrm{CH}_{2}\right), 37.4(\mathrm{CH}), 55.6(2 \mathrm{CH}), 56.1\left(2 \mathrm{CH}_{3}\right), 56.3\left(\mathrm{CH}_{3}\right), 59.8\left(\mathrm{CH}_{3}\right), 60.4\left(\mathrm{CH}_{3}\right), 60.5\left(\mathrm{CH}_{3}\right), 97.7(\mathrm{CH})$, 106.7 (2CH), 108.0 (C), 133.6 (2C), 136.7 (C), 136.9 (C), 152.5 (C), 152.6 (2C), 154.1 (C), 172.6 (C), 174.3 (C) ppm; LC/MS $\left(\mathrm{APCl}^{+}\right) \mathrm{m} / \mathrm{z} 499.22\left(\mathrm{MH}^{+}\right.$) (calcd for $\mathrm{C}_{26} \mathrm{H}_{30} \mathrm{~N}_{2} \mathrm{O}_{8}: 498.54 \mathrm{~g} / \mathrm{mol}$ ).

9,10,11-Trimethoxy-1-\{3,4,5-trimethoxy-2-[5-oxo-1-(3,4,5-trimethoxy-phenyl)pyrrolidin-2-yl]-phenyl\}3a,4,5,11b-tetrahydro-1H-dipyrrolo[1,2-a:3',2'-c]quinoline-2,6(3H,3bH)-dione (15e). This product was obtained as white solid (Method A. Traces; Method B. 24\% yield (0.213 g)); mp (EtOAc/n-heptane) 137-139 ${ }^{\circ} \mathrm{C}$; IR (KBr): v 3525, 3442, 1674, 1594, 1493, 1452, $1105 \mathrm{~cm}^{-1} ;{ }^{1} \mathrm{H}$ NMR $\left(\mathrm{CDCl}_{3}, 400 \mathrm{MHz}\right): \delta 1.85-1.97(\mathrm{~m}, 1 \mathrm{H}$, $\left.\mathrm{CH}_{2} \mathrm{CH}_{2} \mathrm{CH}\right), 2.25-2.40\left(\mathrm{~m}, 2 \mathrm{H}, \mathrm{CH}_{2} \mathrm{CH}_{2} \mathrm{CH}\right), 2.46-2.57\left(\mathrm{~m}, 1 \mathrm{H}, \mathrm{CH}_{2} \mathrm{CH}_{2} \mathrm{CH}\right), 2.58-2.74\left(\mathrm{~m}, 6 \mathrm{H}, 3 \mathrm{CH}_{2}\right), 3.34(\mathrm{~s}, 3 \mathrm{H}$, $\left.\mathrm{OCH}_{3}\right), 3.34-3.43(\mathrm{~m}, 1 \mathrm{H}, \mathrm{CH}), 3.56\left(\mathrm{~s}, 3 \mathrm{H}, \mathrm{OCH}_{3}\right), 3.75(\mathrm{~s}, 6 \mathrm{H}, 2 \mathrm{OCH}), 3.80\left(\mathrm{~s}, 6 \mathrm{H}, 2 \mathrm{OCH}_{3}\right), 3.83(\mathrm{~s}, 6 \mathrm{H}, 2 \mathrm{OCH})$, $3.87\left(\mathrm{~s}, 3 \mathrm{H}, \mathrm{OCH}_{3}\right), 4.01-4.08(\mathrm{~m}, 1 \mathrm{H}, \mathrm{CH}), 5.30(\mathrm{t}, J 7.9 \mathrm{~Hz}, 1 \mathrm{H}, \mathrm{CH}), 5.49(\mathrm{~d}, J 8.9 \mathrm{~Hz}, 1 \mathrm{H}, \mathrm{CH}), 6.17(\mathrm{~s}, 2 \mathrm{H}, \mathrm{ArH})$, $6.41(\mathrm{~s}, 1 \mathrm{H}, \mathrm{ArH}), 7.71(\mathrm{~s}, 1 \mathrm{H}, \mathrm{ArH}) \mathrm{ppm} ;{ }^{13} \mathrm{C} \mathrm{NMR}\left(\mathrm{CDCl}_{3}, 100 \mathrm{MHz}\right): \delta 20.2\left(\mathrm{CH}_{2}\right), 27.3\left(\mathrm{CH}_{2}\right), 30.6\left(\mathrm{CH}_{2}\right), 31.3$ $\left(\mathrm{CH}_{2}\right), 31.6\left(\mathrm{CH}_{2}\right), 38.4(\mathrm{CH}), 55.9\left(\mathrm{CH}_{3}\right), 56.0\left(\mathrm{CH}_{3}\right), 56.1\left(2 \mathrm{CH}_{3}\right), 57.7(\mathrm{CH}), 58.7(\mathrm{CH}), 59.8(\mathrm{CH}), 60.6\left(\mathrm{CH}_{3}\right), 60.7$ $\left(\mathrm{CH}_{3}\right), 60.8\left(2 \mathrm{CH}_{3}\right), 61.3\left(\mathrm{CH}_{3}\right), 98.7(\mathrm{CH}), 105.1(2 \mathrm{CH}), 108.1(\mathrm{CH}), 111.6(\mathrm{C}), 124.9(\mathrm{C}), 130.8(\mathrm{C}), 133.6(\mathrm{C})$, 133.9 (C), 137.2 (C), 137.6 (C), 142.2 (C), 151.5 (C), 152.3 (C), 153.2 (2C), 153.6 (C), 153.9 (C), 172.7 (C), 173.1 (C), 175.7 (C) ppm; LC/MS $\left(\mathrm{APCl}^{+}\right) \mathrm{m} / \mathrm{z} 748.33\left(\mathrm{MH}^{+}\right)$(calcd for $\mathrm{C}_{39} \mathrm{H}_{45} \mathrm{~N}_{3} \mathrm{O}_{12}: 747.81 \mathrm{~g} / \mathrm{mol}$ ).

5-Hydroxy-1-(3,4,5-trimethoxyphenyl)pyrrolidin-2-one (19e). This product was obtained as yellow-brownish oil with Method A in 40\% yield $(0.380 \mathrm{~g})$. It was also obtained using Method B in only $5 \%$ yield $(0.048 \mathrm{~g})$; IR (KBr): v 3344, 1671, 1591, 1504, 1451, $1121 \mathrm{~cm}^{-1} ;{ }^{1} \mathrm{H}$ NMR $\left(\mathrm{CDCl}_{3}, 400 \mathrm{MHz}\right): \delta 1.99-2.09\left(\mathrm{~m}, 1 \mathrm{H}, \mathrm{CH}_{2} \mathrm{CH}_{2} \mathrm{CH}\right)$, 2.35-2.48 (m, $\left.2 \mathrm{H}, \mathrm{CH}_{2} \mathrm{CH}_{2} \mathrm{CH}\right), 2.68-2.78\left(\mathrm{~m}, 1 \mathrm{H}, \mathrm{CH}_{2} \mathrm{CH}_{2} \mathrm{CH}\right), 3.78\left(\mathrm{~s}, 3 \mathrm{H}, \mathrm{OCH}_{3}\right), 3.79(\mathrm{~s}, 6 \mathrm{H}, 2 \mathrm{OCH}), 3.79$ (bs,

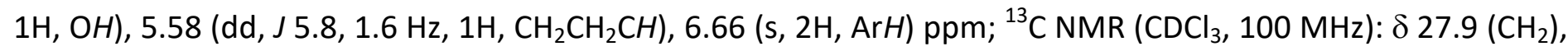
$29.7\left(\mathrm{CH}_{2}\right), 56.1\left(2 \mathrm{CH}_{3}\right), 60.8\left(\mathrm{CH}_{3}\right), 85.5(\mathrm{CH}), 102.3(2 \mathrm{CH}), 132.9(\mathrm{C}), 136.1(\mathrm{C}), 153.2(2 \mathrm{C}), 174.6(\mathrm{C}) \mathrm{ppm}$; $\mathrm{LC} / \mathrm{MS}\left(\mathrm{APCl}^{+}\right) \mathrm{m} / \mathrm{z} 268.10\left(\mathrm{MH}^{+}\right)$(calcd for $\mathrm{C}_{13} \mathrm{H}_{17} \mathrm{NO}_{5}: 267.28 \mathrm{~g} / \mathrm{mol}$ ).

Reaction of $\mathbf{N}$-(4-nitrophenyl)pterolactam 1 f with TMSOTf. The following compounds were obtained by us sing Method B N-(4-nitrophenyl)pterolactam (1f, $1.0 \mathrm{~g}, 4.23 \mathrm{mmol})$ and TMSOTf $(0.5 \mathrm{~mL}, 0.61 \mathrm{~g}, 2.7 \mathrm{mmol}) \mathrm{in}$ $10 \mathrm{~mL}$ of $\mathrm{CH}_{2} \mathrm{Cl}_{2}$ were stirred at $\mathrm{rt}$ for $1.5 \mathrm{~h}$.

1-(4-Nitrophenyl)-4-(1-(4-nitrophenyl)-5-oxopyrrolidin-2-yl)-1H-pyrrol-2(5H)-one (17f). This product was obtained as cream solid; $25 \%$ yield (0.43 g); mp (EtOAc/ $n$-heptane) $149-151{ }^{\circ} \mathrm{C}$; IR (KBr): v 1711, 1593, 1498, 1156, $1112 \mathrm{~cm}^{-1} ;{ }^{1} \mathrm{H}$ NMR (DMSO-d $\left.6,400 \mathrm{MHz}\right): \delta 2.15-2.26\left(\mathrm{~m}, 1 \mathrm{H}, \mathrm{CH}_{2} \mathrm{CH}_{2} \mathrm{CH}\right), 2.55-2.67\left(\mathrm{~m}, 2 \mathrm{H}, \mathrm{CH}_{2} \mathrm{CH}_{2} \mathrm{CH}_{\text {) }}\right.$, 2.72-2.85 (m, 1H, $\left.\mathrm{CH}_{2} \mathrm{CH}_{2} \mathrm{CH}\right), 4.65\left(\mathrm{dd}, J\right.$ 19.4, $\left.1.6 \mathrm{~Hz}, 1 \mathrm{H}, \mathrm{CH}_{2}\right), 4.90\left(\mathrm{dd}, J 19.4,1.6 \mathrm{~Hz}, 1 \mathrm{H}, \mathrm{CH}_{2}\right), 5.56(\mathrm{dd}, J$ 8.2, 3.1 Hz, $1 \mathrm{H}, \mathrm{CH}$ ), $6.02(\mathrm{dt}, J$ 1.6, $1.0 \mathrm{~Hz}, 1 \mathrm{H}, \mathrm{CH}$ ), 7.90 (dd, J 9.4, 2.4 Hz, 2H, ArH), 7.97 (dd, J 9.4, 2.4 Hz, 2H, $\operatorname{ArH}$ ), 8.24 (dd, J 9.4, $2.4 \mathrm{~Hz}, 2 \mathrm{H}, \mathrm{ArH}), 8.27$ (dd, J 9.4, $2.4 \mathrm{~Hz}, 2 \mathrm{H}, \mathrm{ArH}$ ) ppm; ${ }^{13} \mathrm{C}$ NMR (DMSO- $\left.d_{6}, 100 \mathrm{MHz}\right): \delta$ $24.6\left(\mathrm{CH}_{2}\right), 31.2\left(\mathrm{CH}_{2}\right), 52.3(\mathrm{CH}), 57.9(\mathrm{CH}), 118.0(2 \mathrm{CH}), 121.1(2 \mathrm{CH}), 123.4(\mathrm{CH}), 125.0(2 \mathrm{CH}), 125.4(2 \mathrm{CH})$, 142.6 (C), 143.4 (C), 144.3 (C), 145.2 (C), 160.9 (C), 170.0 (C), 175.1 (C) ppm; Anal. Calcd for $\mathrm{C}_{20} \mathrm{H}_{16} \mathrm{~N}_{4} \mathrm{O}_{6}: \mathrm{C}_{\text {, }}$ 58.82; H, 3.95; N, 13.72. Found: C, 59.24; H, 4.31; N, $14.01 \%$.

1-(4-Nitrophenyl)-3-(1-(4-nitrophenyl)-5-oxopyrrolidin-2-yl)-1H-pyrol-2(5H)-one (18f). This product was obtained as cream solid; $5 \%$ yield $(0.043 \mathrm{~g})$; $\mathrm{mp}$ (EtOAc/ $n$-heptane) $137-139{ }^{\circ} \mathrm{C}$; IR (KBr): v 1716, 1682, 1591, 1493, 1442, 1194, 1156, 1111, $1067 \mathrm{~cm}^{-1} ;{ }^{1} \mathrm{H}$ NMR (DMSO- $\left.d_{6}, 400 \mathrm{MHz}\right): \delta 2.01-2.12\left(\mathrm{~m}, 1 \mathrm{H}, \mathrm{CH}_{2} \mathrm{CH}_{2} \mathrm{CH}\right.$ ), $2.53-$ 
$2.64\left(\mathrm{~m}, 2 \mathrm{H}, \mathrm{CH}_{2} \mathrm{CH}_{2} \mathrm{CH}\right), 2.69-2.84\left(\mathrm{~m}, 1 \mathrm{H}, \mathrm{CH}_{2} \mathrm{CH}_{2} \mathrm{CH}\right), 4.56$ (dd, J 20.9, $\left.1.2 \mathrm{~Hz}, 2 \mathrm{H}, \mathrm{CH}_{2}\right), 5.44(\mathrm{~d}, J 7.3 \mathrm{~Hz}, 1 \mathrm{H}$, $\mathrm{CH}$ ), 7.26 (dd, J 2.2, 1.2 Hz, 1H, CH), 7.89 (dd, J 9.5, 1.6 Hz, 2H, ArH), $8.03(\mathrm{dd}, J$ 9.5, $1.6 \mathrm{~Hz}, 2 \mathrm{H}, \operatorname{ArH}), 8.23(\mathrm{dd}, J$ 9.4, $1.5 \mathrm{~Hz}, 2 \mathrm{H}, \mathrm{ArH}), 8.28$ (dd, J 9.4, $1.5 \mathrm{~Hz}, 2 \mathrm{H}, \mathrm{ArH}) \mathrm{ppm} ;{ }^{13} \mathrm{C}$ NMR (DMSO-d, $\left.100 \mathrm{MHz}\right) \delta(\mathrm{ppm}) 24.3\left(\mathrm{CH}_{2}\right)$, $31.3\left(\mathrm{CH}_{2}\right), 51.7(\mathrm{CH}), 55.9(\mathrm{CH}), 118.2(2 \mathrm{CH}), 121.1(2 \mathrm{CH}), 124.9(2 \mathrm{CH}), 125.4(2 \mathrm{CH}), 137.4(\mathrm{CH}), 141.4(\mathrm{C})$, 142.9 (C), 143.3 (C), 144.5 (C), 145.1 (C), 169.1 (C), 175.4 (C) ppm; Anal. Calcd for $\mathrm{C}_{20} \mathrm{H}_{16} \mathrm{~N}_{4} \mathrm{O}_{6}$ : C, 58.82; $\mathrm{H}, 3.95$; $N, 13.72$. Found: $C, 59.07 ; H, 4.15 ; N, 13.99 \%$.

Reaction of $\mathbf{N}$-(biphenyl)pterolactam $\mathbf{1 g}$ with TMSOTf. The following compounds were obtained by us sing Method B: $N$-(Biphenyl)pterolactam (1g, $0.8 \mathrm{~g}, 2.99 \mathrm{mmol})$ and TMSOTf $(0.4 \mathrm{~mL}, 0.49 \mathrm{~g}, 2.2 \mathrm{mmol})$ in $10 \mathrm{~mL}$ of $\mathrm{CH}_{2} \mathrm{Cl}_{2}$ were stirred at $\mathrm{rt}$ for $1.5 \mathrm{~h}$. Trimers were not stable enough in deuterated solvents to conduct additional experiments to elucidate the exact stereochemical of the trimeric products.

5,5'-(1-([1,1'-Biphenyl]-4-yl)-2-oxo-2,3-dihydro-1H-pyrrole-3,4-diyl)bis(1-([1,1'-biphenyl]-4-yl)-pyrrolidin-2one) (16g). This product was obtained as white solid; $10 \%$ yield $(0.070 \mathrm{~g}) ; \mathrm{mp}$ (EtOAc/n-heptane) $259-262{ }^{\circ} \mathrm{C}$; IR (KBr): v 1697, 1606, 1521, 1487, 1156, $1081 \mathrm{~cm}^{-1} ;{ }^{1} \mathrm{H}$ NMR $\left(\mathrm{CDCl}_{3}, 400 \mathrm{MHz}\right): \delta$ 0.91-1.05 $\left(\mathrm{m}, 1 \mathrm{H}, \mathrm{CH}_{2} \mathrm{CH}_{2} \mathrm{CH}\right)$, 1.20-1.29 (m, 3H, $\left.\mathrm{CH}_{2} \mathrm{CH}_{2} \mathrm{CH}\right), 1.63-1.68\left(\mathrm{~m}, 1 \mathrm{H}, \mathrm{CH}_{2} \mathrm{CH}_{2} \mathrm{CH}\right), 1.94-2.01\left(\mathrm{~m}, 2 \mathrm{H}, \mathrm{CH}_{2} \mathrm{CH}_{2} \mathrm{CH}\right), 2.63-2.74(\mathrm{~m}, 3 \mathrm{H}$, $\left.\mathrm{CH}_{2} \mathrm{CH}_{2} \mathrm{CH}\right), 4.56-4.62(\mathrm{~m}, 1 \mathrm{H}, \mathrm{CH}), 4.97(\mathrm{~d}, J 2.4 \mathrm{~Hz}, 1 \mathrm{H}, \mathrm{CH}), 5.46-5.51(\mathrm{~m}, 1 \mathrm{H}, \mathrm{CH}), 6.70(\mathrm{~d}, J 1.2 \mathrm{~Hz}, 1 \mathrm{H}, \mathrm{CH})$,

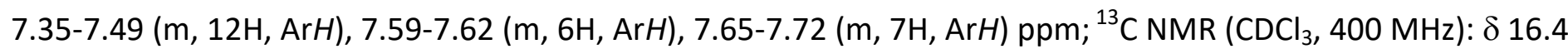
$\left(\mathrm{CH}_{2}\right), 25.5\left(\mathrm{CH}_{2}\right), 29.8\left(\mathrm{CH}_{2}\right), 31.2\left(\mathrm{CH}_{2}\right), 55.4(\mathrm{CH}), 57.8(\mathrm{CH}), 61.2(\mathrm{CH}), 121.9(2 \mathrm{CH}), 122.7(2 \mathrm{CH}), 123.6(2 \mathrm{CH})$, $127.0(6 \mathrm{CH}), 127.5(\mathrm{CH}), 127.7(2 \mathrm{CH}), 128.0(2 \mathrm{CH}), 128.2(2 \mathrm{CH}), 128.3(2 \mathrm{CH}), 128.9(6 \mathrm{CH}), 134.8(\mathrm{C}), 135.6(\mathrm{C})$, 136.2 (C), 136.9 (C), 138.6 (C), 139.2 (C), 139.5 (C), 140.0 (2C), 140.1 (C), 142.7 (C), 168.0 (C), 173.4 (C), 174.2 (C) ppm.

5,5'-(1-([1,1'-Biphenyl]-4-yl)-5-oxo-2,5-dihydro-1H-pyrrole-2,4-diyl)bis(1-([1,1'-biphenyl]-4-yl)pyrrolidin-2one) (16'g). This product was obtained as white solid; $8 \%$ yield (0.056 g); mp (EtOAc/n-heptane) $175-178{ }^{\circ} \mathrm{C}$; IR $(\mathrm{KBr})$ : v 1689, 1605, 1521, 1486, 1156, $1032 \mathrm{~cm}^{-1} ;{ }^{1} \mathrm{H} \mathrm{NMR}\left(\mathrm{CDCl}_{3}, 400 \mathrm{MHz}\right): \delta$ 0.77-0.84 $\left(\mathrm{m}, 1 \mathrm{H}, \mathrm{CH}_{2} \mathrm{CH}_{2} \mathrm{CH}\right)$, 1.61-1.67 (m, $\left.1 \mathrm{H}, \mathrm{CH}_{2} \mathrm{CH}_{2} \mathrm{CH}\right), 1.93-2.01\left(\mathrm{~m}, 1 \mathrm{H}, \mathrm{CH}_{2} \mathrm{CH}_{2} \mathrm{CH}\right), 2.18-2.23\left(\mathrm{~m}, 3 \mathrm{H}, \mathrm{CH}_{2} \mathrm{CH}_{2} \mathrm{CH}\right), 2.57-2.66(\mathrm{~m}, 3 \mathrm{H}$, $\left.\mathrm{CH}_{2} \mathrm{CH}_{2} \mathrm{CH}\right), 2.90-3.01\left(\mathrm{~m}, 1 \mathrm{H}, \mathrm{CH}_{2} \mathrm{CH}_{2} \mathrm{CH}\right), 4.53-4.59(\mathrm{~m}, 1 \mathrm{H}, \mathrm{CH}), 4.93(\mathrm{~d}, \mathrm{~J} 2.4 \mathrm{~Hz}, 1 \mathrm{H}, \mathrm{CH}), 5.25-5.29(\mathrm{~m}, 1 \mathrm{H}$, $\mathrm{CH}), 6.86(\mathrm{~d}, \mathrm{~J} 1.2 \mathrm{~Hz}, 1 \mathrm{H}, \mathrm{CH}), 7.31-7.38(\mathrm{~m}, 3 \mathrm{H}, \mathrm{ArH}), 7.41-7.48(\mathrm{~m}, 9 \mathrm{H}, \mathrm{ArH}), 7.52-7.68(\mathrm{~m}, 13 \mathrm{H}, \mathrm{ArH}) \mathrm{ppm} ;{ }^{13} \mathrm{C}$ NMR $\left(\mathrm{CDCl}_{3}, 100 \mathrm{MHz}\right): \delta 16.4\left(\mathrm{CH}_{2}\right), 23.5\left(\mathrm{CH}_{2}\right), 30.9\left(\mathrm{CH}_{2}\right), 31.6\left(\mathrm{CH}_{2}\right), 39.4\left(\mathrm{CH}_{2}\right), 40.6(\mathrm{CH}), 56.7(\mathrm{CH}), 57.7$ $(\mathrm{CH}), 60.8(\mathrm{CH}), 123.4(\mathrm{CH}), 124.4(2 \mathrm{CH}), 124.6(\mathrm{CH}), 126.9(3 \mathrm{CH}), 127.0(3 \mathrm{CH}), 127.1(3 \mathrm{CH}), 127.3(2 \mathrm{CH}), 127.7$ $(2 \mathrm{CH}), 127.8(\mathrm{CH}), 127.9(\mathrm{CH}), 129.4(6 \mathrm{CH}), 135.9(\mathrm{C}), 136.6(\mathrm{C}), 137.4(\mathrm{C}), 137.5(\mathrm{C}), 137.9(\mathrm{C}), 138.0(\mathrm{C}), 139.7$ (2C), 139.8 (2C), 139.9 (C), 140.5 (C), 140.8 (C), 167.8 (C), 173.6 (C), 174.6 (C) ppm.

1-(1,1'-Biphenyl-4-yl)-3-[1-(1,1'-biphenyl-4-yl)-5-oxo-pyrrolidin-2-yl]-1,5-dihydro-2H-pyrrol-2-one (18g). This product was obtained as white solid; $7 \%$ yield (0.049 g); $\mathrm{mp}$ (EtOAc/n-heptane) 257-260 ${ }^{\circ} \mathrm{C} ; \mathrm{IR}(\mathrm{KBr}): \mathrm{v} 1677$, 1609, 1523, 1485, 1151, $1091 \mathrm{~cm}^{-1} ;{ }^{1} \mathrm{H}$ NMR $\left(\mathrm{CDCl}_{3}, 400 \mathrm{MHz}\right): \delta 2.14-2.21$ (sym m, $1 \mathrm{H}, \mathrm{CH}_{2} \mathrm{CH}_{2} \mathrm{CH}$ ), $2.59-2.72$ (m, $\left.2 \mathrm{H}, \mathrm{CH}_{2} \mathrm{CH}_{2} \mathrm{CH}\right), 2.72-2.80\left(\mathrm{~m}, 1 \mathrm{H}, \mathrm{CH}_{2} \mathrm{CH}_{2} \mathrm{CH}\right), 4.37\left(\mathrm{t}, J 1.8 \mathrm{~Hz}, 2 \mathrm{H}, \mathrm{CH}_{2}\right), 5.33(\mathrm{bd}, J 5.7 \mathrm{~Hz}, 1 \mathrm{H}, \mathrm{CH}), 6.79$ (dd, J 3.3, 1.8 Hz, 1H, CH), 7.30-7.37 (m, 2H, ArH), 7.40-7.47 (m, 4H, ArH), 7.54-7.67 (m, 10H, ArH), 7.80 (d, J $8.8 \mathrm{~Hz}, 2 \mathrm{H}, \mathrm{ArH}) \mathrm{ppm} ;{ }^{13} \mathrm{C} \mathrm{NMR}\left(\mathrm{CDCl}_{3}, 100 \mathrm{MHz}\right): \delta 25.0\left(\mathrm{CH}_{2}\right), 31.1\left(\mathrm{CH}_{2}\right), 51.2\left(\mathrm{CH}_{2}\right), 56.3(\mathrm{CH}), 118.8(2 \mathrm{CH})$, $121.5(2 \mathrm{CH}), 126.8(2 \mathrm{CH}), 126.9(2 \mathrm{CH}), 127.2(\mathrm{CH}), 127.5(\mathrm{CH}), 127.6(2 \mathrm{CH}), 127.7(2 \mathrm{CH}), 128.8(2 \mathrm{CH}), 128.9$ $(2 \mathrm{CH}), 135.8(2 \mathrm{C}), 137.1(\mathrm{CH}), 137.3$ (C), 137.7 (C), 138.0 (C), 140.0 (C), 140.2 (C), 168.3 (C), 174.5 (C) ppm; Anal. Calcd for $\mathrm{C}_{32} \mathrm{H}_{26} \mathrm{~N}_{2} \mathrm{O}_{2}: \mathrm{C}, 81.68 ; \mathrm{H}, 5.57 ; \mathrm{N}, 5.95$. Found: $\mathrm{C}, 81.81 ; \mathrm{H}, 5.72 ; \mathrm{N}, 6.30 \%$.

1-(1,1'-Biphenyl-4-yl)-5-hydroxypyrrolidin-2-one (19g). This product was obtained as white solid; $2 \%$ yield (0.015 g); $\mathrm{mp}$ (EtOAc/n-heptane) 278-180 ${ }^{\circ} \mathrm{C}$; IR (KBr): v 3218, 1661, 1607, 1522, 1485, 1452, 1166, 1123, 1093 $\mathrm{cm}^{-1} ;{ }^{1} \mathrm{H}$ NMR $\left(\mathrm{CDCl}_{3}, 400 \mathrm{MHz}\right): \delta$ 2.00-2.09 (m, $\left.1 \mathrm{H}, \mathrm{CH}_{2} \mathrm{CH}_{2} \mathrm{CH}\right), 2.36-2.52\left(\mathrm{~m}, 2 \mathrm{H}, \mathrm{CH}_{2} \mathrm{CH}_{2} \mathrm{CH}\right), 2.71-2.83(\mathrm{~m}$, $\left.1 \mathrm{H}, \mathrm{CH}_{2} \mathrm{CH}_{2} \mathrm{CH}\right), 3.43(\mathrm{~d}, J 6.9 \mathrm{~Hz}, 1 \mathrm{H}, \mathrm{OH}), 5.67(\mathrm{td}, J 6.4,1.8 \mathrm{~Hz}, 1 \mathrm{H}, \mathrm{CH}), 7.33(\mathrm{ddt}, J$ 8.1, 6.6, 1.4 Hz, 1H, $\mathrm{ArH})$, $7.41(\mathrm{tt}, J$ 7.5, $1.5 \mathrm{~Hz}, 2 \mathrm{H}, \mathrm{ArH}), 7.52-7.56(\mathrm{~m}, 2 \mathrm{H}, \mathrm{ArH}), 7.58(\mathrm{~s}, 4 \mathrm{H}, \mathrm{ArH}) \mathrm{ppm} ;{ }^{13} \mathrm{C} \mathrm{NMR}\left(\mathrm{CDCl}_{3}, 100 \mathrm{MHz}\right) \delta$ 
(ppm) $28.2\left(\mathrm{CH}_{2}\right), 29.7\left(\mathrm{CH}_{2}\right), 85.1(\mathrm{CH}), 123.6(2 \mathrm{CH}), 127.0(2 \mathrm{CH}), 127.4(\mathrm{CH}), 127.8(2 \mathrm{CH}), 128.8(2 \mathrm{CH}), 136.3$ (C), 139.0 (C), 140.3 (C), 174.4 (C) ppm; Anal. Calcd for $\mathrm{C}_{16} \mathrm{H}_{15} \mathrm{NO}_{2}: \mathrm{C}, 75.87 ; \mathrm{H}, 5.97$; N, 5.53. Found: C, 76.02; $\mathrm{H}, 6.11 ; \mathrm{N}, 5.90 \%$.

1-(1,1'-Biphenyl-4-yl)-1,5-dihydro-2H-pyrrol-2-one (20g). This product was obtained as white solid; $2 \%$ yield (0.014 g); mp (EtOAc/n-heptane) 278-180 ${ }^{\circ} \mathrm{C}$; IR (KBr): v 1672, 1601, 1525, 1488, 1453, 1147, $1073 \mathrm{~cm}^{-1} ;{ }^{1} \mathrm{H}$ $\mathrm{NMR}\left(\mathrm{CDCl}_{3}, 400 \mathrm{MHz}\right): \delta 4.64\left(\mathrm{td}, J 2.0,0.8 \mathrm{~Hz}, 2 \mathrm{H}, \mathrm{CH}_{2}\right), 6.27(\mathrm{dt}, J 6.1,2.0 \mathrm{~Hz}, 1 \mathrm{H}, \mathrm{CH}), 7.35(\mathrm{t}, J 7.4 \mathrm{~Hz}, 1 \mathrm{H}$, $\mathrm{CH}$ ), $7.46(\mathrm{t}, J 7.7 \mathrm{~Hz}, 2 \mathrm{H}, \mathrm{ArH}), 7.49(\mathrm{dt}, J 6.1,2.0 \mathrm{~Hz}, 1 \mathrm{H}, \mathrm{CH}), 7.68(\mathrm{dd}, J 7.3,0.9 \mathrm{~Hz}, 2 \mathrm{H}, \operatorname{ArH}), 7.70(\mathrm{dd}, J 9.0$,

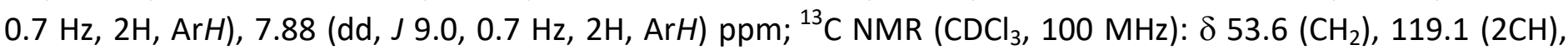
$126.8(2 \mathrm{CH}), 127.5(3 \mathrm{CH}), 128.0(\mathrm{CH}), 129.4(2 \mathrm{CH}), 135.6(\mathrm{C}), 139.2(\mathrm{C}), 140.0(\mathrm{C}), 145.7(\mathrm{CH}), 170.3(\mathrm{C}) \mathrm{ppm}$; Anal. Calcd for $\mathrm{C}_{16} \mathrm{H}_{13} \mathrm{NO}$ : C, 81.68; $\mathrm{H}, 5.57 ; \mathrm{N}, 5.95$. Found: $\mathrm{C}, 81.73 ; \mathrm{H}, 5.80 ; \mathrm{N}, 6.08 \%$.

\section{Acknowledgements}

The authors are grateful to HEl, Yncréa Hauts-de-France and the scientific council of the University of Le Havre Normandie from 'Normandie Université, France' for their financial support and technical helps. We also addressed special thanks to 'Al. I. Cuza' University of lasi (Romania) for all facilities given to one of us G.-M. Dumitriu.

\section{References}

1. Speckamp, W. N.; Moolenaar, M. J. Tetrahedron 56, 2000, 3817-3856.

https://doi.org/10.1016/S0040-4020(00)00159-9

2. Marson, C. M. Arkivoc 2001, i, 1-16.

https://doi.org/10.3998/ark.5550190.0002.101

3. Maryanoff, B. E.; Zhang, H. C.; Cohen, J. H.; Turchi, I. J.; Maryanoff, C. A. Chem. Rev. 2004, 104, 14311628.

https://doi.org/10.1021/cr0306182

4. Yazici, A.; Pyne, S. G. Synthesis 2009, 3, 339-368.

https://doi.org/10.1055/s-0028-1083325

5. Yazici, A.; Pyne, S. G. Synthesis 2009, 4, 513-541.

https://doi.org/10.1055/s-0028-1083346

6. Wu, P.; Nielsen, T. E. Chem. Rev. 2017, 117, 7811-7856.

https://doi.org/10.1021/acs.joc.0c00482

7. Daïch, A.; Ghinet, A.; Rigo, B. Addition to N-Acyliminium Ions of Heteroatoms such as Oxygen, Nitrogen, Sulfur, and Selenium as Internal Nucleophiles. In Comprehensive Organic Synthesis, Molander, G.A., Knochel, P., Eds., Oxford: Elsevier, 2014, $2^{\text {nd }}$ Edition, Vol. 2, Chap. 2.21, pp 682-742.

https://doi.org/10.1016/B978-0-08-097742-3.00221-4

8. Chihab-Eddine, A.; Daïch, A.; Jilale, A.; Decroix, B. Tetrahedron Lett. 2001, 42, 573-576. https://doi.org/10.1016/S0040-4039(00)02037-2

9. Ghinet, A.; Abuhaie, C.-M.; Homerin, G.; Marzag, H.; Dubois, J.; Rigo, B.; Daïch, A. ChemistrySelect 2017, 2, 10654-10660.

https://doi.org/10.1002/slct.201701766 
10. Winter, C.; Pullin, R. D. C.; Donohoe, T. J. Tetrahedron Lett. 2017, 58, 602-605. https://doi.org/10.1016/j.tetlet.2016.12.045

11. Šafář, P.; Marchalín, Š.; Prónayová, N.; Vrábel, V.; Lawson, A. M.; Othman, M.; Daïch, A. Tetrahedron 2016, 72, 3221-3231.

https://doi.org/10.1016/i.tet.2016.04.047

12. Comesse, S.; Sanselme, M.; Daïch, A. J. Org. Chem. 2008, 73, 5566-5569. https://doi.org/10.1021/jo702752w

13. Rigo, B.; Akué-Gédu, R. Targets Heterocyclic Syst. 2006, 10, 232-265.

14. Rigo, B.; Cauliez, P.; Fasseur, D.; Sauvage, F. X.; Trends Heterocyclic Chem. 1991, 2, 155-204.

15. Dumitriu, G. M.; Bîcu, E.; Eryuruk, U.; Belei, D.; Rigo, B.; Daïch, A.; Ghinet, A. Synlett 2016, 27, $934-940$. http://dx.doi.org/10.1055/s-0035-1561333

16. Einsiedel, J.; Hubner, H.; Gmeiner, P. Bioorg. Med. Chem. Lett. 2003, 13, 851-854. https://doi.org/10.1016/S0960-894X(03)00004-0

17. Kosugi, Y.; Nagasaka, T.; Hamaguchi, F.; Ohki, S. Heterocycles 1986, 24, 625-628. https://doi.org/10.3987/R-1986-03-0625

18. Fabre, J. L.; Farge, D.; James, C.; Lave, D. Rhone-Poulenc Santé. EP 110,795; (1984) Chem Abstr 101, 110726.

19. Baussanne, I.; Dudot, B.; Perard-Viret, J.; Planas, L.; Royer, J. Arkivoc 2006, vii, 57-66. https://doi.org/10.3998/ark.5550190.0007.706

20. Shono, T.; Matsumura, Y.; Tsubata, K.; Sugihara, Y. Tetrahedron Lett. 1982, 23, 1201-1204. https://doi.org/10.1016/S0040-4039(00)87059-8

21. Nyberg, K. Synthesis 1976, 8, 545-546. https://doi.org/10.1055/s-1976-24118

22. Wijnberg, J. B. P. A.; Speckamp, W. N.; Schoemaker, H. E. Tetrahedron Lett. 1974, 15, 4073-4076. https://doi.org/10.1016/S0040-4039(01)92087-8

23. Wijnberg, J. B. P. A.; Speckamp, W. N.; DeBoer, J. J. J. Tetrahedron Lett. 1974, 15, 4077-4080. https://doi.org/10.1016/S0040-4039(01)92088-X

24. Zhang, W.; Dai, Y.; Wang, X.; Zhang, W. Tetrahedron Lett. 2011, 52, 6122-6126. https://doi.org/10.1016/j.tetlet.2011.09.021

25. Jia, X.; Han, B.; Zhang, W.; Jin, X.; Yang, L.; Liu, Z. L. Synthesis 2006, 17, 2831-2836. https://doi.org/10.1055/s-2006-942521

26. Jia, X. D.; Ren, Y.; Huo, C. D.; Wang, W. J.; Chen, X. N.; Xu, X. L.; Wang, X. C. Tetrahedron Lett. 2010, 51, 6779-6782.

https://doi.org/10.1016/j.tetlet.2010.10.106

27. Tanaka, S.; Yasuda, M.; Baba, A. J. Org. Chem. 2006, 71, 800-803. https://doi.org/10.1021/jo052004y

28. Povarov, L. S. Russ. Chem. Rev. 1967, 36, 656-670. https://doi.org/10.1070/RC1967v036n09ABEH001680

29. Kouznetsov, V. V. Tetrahedron 2009, 65, 2721-2750. https://doi.org/10.1016/i.tet.2008.12.059

30. Sundararajan, G.; Prabagaran, N.; Varghese, B. Org. Lett. 2001, 3, 1973-1976. https://doi.org/10.1021/ol0159221

31. Shachkus, A. A.; Degutis, Y. A.; Mikul'skis, P. P. Chem. Heterocycl. Compd. 1989, 25, 47-50. https://doi.org/10.1007/BF00472617 
32. Speckamp, W. N.; DeBoer, J. J. J. Recl. Trav. Chim. Pays-Bas 1983, 102, 405-409. https://doi.org/10.1002/recl.19831020905

33. Speckamp, W. N.; DeBoer, J. J. J. Recl. Trav. Chim. Pays-Bas 1983, 102, 410-414. https://doi.org/10.1002/recl.19831020906

34. Valuer, R. É.; Karlivan, G. A. Chem. Heterocycl. Compd. 1976, 12, 999-1002. https://doi.org/10.1007/BF00480391

35. Baudelet, D.; Daïch, A.; Rigo, B.; Lipka, E.; Gautret, P.; Homerin, G.; Claverie, C.; Rousseau, J.; Abuhaie, C. M.; Ghinet, A. Synthesis 2016, 48, 2226-2244.

https://doi.org/10.1055/s-0035-1561415

36. Gephart, III., R. T.; Huang, D. L.; Aguila, M. J. B.; Schmidt, G.; Shahu, A.; Warren, T. H.; Angew. Chem. Int. Edit. 2012, 51, 6488-6492.

https://doi.org/10.1002/anie.201201921

37. Chen, Y. W.; Chen, Y. L.; Tseng, C. H.; Liang, C. C.; Yang, C. N.; Yao, Y. C.; Lu, P. J.; Tzeng, C. C. J. Med. Chem. 2011, 54, 4446-4461.

https://doi.org/10.1021/im200046z

38. Sabatini, S.; Gosetto, F.; Manfroni, G.; Tabarrini, O.; Kaatz, G. W.; Patel, D.; Cecchetti, V. J. Med. Chem. 2011, 54, 5722-5736.

https://doi.org/10.1021/im200370y

39. Lilienkampf, A.; Mao, J.; Wan, B.; Wang, Y.; Franzblau, S. G.; Kozikowski, A. P. J. Med. Chem. 2009, 52, 2109-2118.

https://doi.org/10.1021/jm900003c

40. Michael, J. P. Nat. Prod. Rep. 2008, 25, 166-187.

https://doi.org/10.1039/B612168N

41. Levy, S.; Azoulay, S. J. Cardiovas. Electrophysiol. 1994, 5, 635-636.

https://doi.org/10.1111/j.1540-8167.1994.tb01304.x

42. Wenckebach, K. F. JAMA 1923, 81, 472-474.

https://doi.org/10.1001/jama.1923.02650060042012

43. Marco-Contelles, J.; Perez-Mayoral, E.; Samadi, A.; Carreiras, M. C.; Soriano, E. Chem. Rev. 2009, 109, 2652-2671.

https://doi.org/10.1021/cr800482c

44. Kouznetsov, V. V.; Mendez, L. Y. V.; Gomez, C. M. M. Curr. Org. Chem. 2005, 9, 141-146. https://doi.org/10.2174/1385272053369196

45. Pesquet, A.; Daïch, A.; Coste, S.; Van Hijfte, L. Synthesis 2008, 9, 1389-1396. https://doi.org/10.1055/s-2008-1067032

46. Fleury, J. F.; Netchitaïlo, P.; Daïch, A. Synlett 2011, 13, 1821-1826. https://doi.org/10.1055/s-0030-1260949

47. Ali, B.; Zukerman-Schpector, J.; Ferreira, F. P.; Shamim, A.; Pimenta, D. C.; Stefani, H. A. Tetrahedron Lett. 2015, 56, 1153-1158.

https://doi.org/10.1016/j.tetlet.2015.01.059

48. Qiao, L.; Zhou, Y.; Zhang, W. Chin. J. Chem. 2010, 28, 449-456.

https://doi.org/10.1002/cjoc.201090095

49. Zhang, W.; Zheng, A.; Liu, Z.; Yang, L.; Liu, Z. Tetrahedron Lett. 2005, 46, 5691-5694. https://doi.org/10.1016/j.tetlet.2005.06.097 
50. Zhang, W.; Huang, L.; Wang, J. Synthesis 2006, 12, 2053-2063.

https://doi.org/10.1055/s-2006-942372

51. Hubert, J. C.; Wijnberg, J. B. P. A.; Speckamp, W. N. Tetrahedron 1975, 31, 1437-1441. https://doi.org/10.1016/0040-4020(75)87076-1

52. Wijnberg, J. B. P. A.; DeBoer, J. J. J.; Speckamp, W. N. Recl. Trav. Chim. Pays-Bas 1978, 97, $227-231$. https://doi.org/10.1002/recl.19780970903

53. Schoemaker, H. E.; Boer-Terpstra, T.; Dijkink, J.; Speckamp, W. N. Tetrahedron 1980, 36, $143-148$. https://doi.org/10.1016/0040-4020(80)85036-8

54. Crystallographic data for products $14 \mathrm{e}-\alpha$ and $14 \mathrm{e}-\beta$ have been deposited at the Cambridge Crystallographic Data Centre (respectively CCDC 1515046 and CCDC 1946958). Copies of all data can be obtained free of charge via the following link:

http://www.ccdc.cam.ac.uk

55. Brown, P. D.; Willis, A. C.; Sherburn, M. S.; Lawrence, A. L. Angew. Chem. Int. Ed. 2013, 52, $13273-13275$. The structure proposed initially as indolo[1.7]naphthyridine with a 6-5-6-fused skeleton for Incargranine-B was revised into 5-6-5-fused skeleton.

https://doi.org/10.1002/anie.201307875

56. Liu, L.; Wang, C.; Liu, Q.; Kong, Y.; Chang, W.; Li, J. Eur. J. Org. Chem. 2016, 3684-3690.

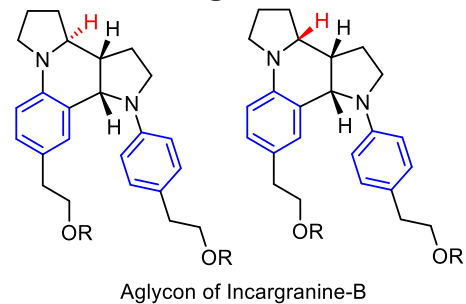

https://doi.org/10.1002/ejoc.201600692

57. Cepanec, I.; Litvić, M.; Udiković, J.; Pogorelić, I.; Lovrić, M. Tetrahedron 2007, 63, 5614-5621. https://doi.org/10.1016/i.tet.2007.04.016 